\title{
Fast switching of mesoscopic magnets
}

\author{
Thomas Schrefl, Josef Fidler, Rok Dittrich, Dieter Suess, Werner Scholz, \\ Vassilios Tsiantos, and Hermann Forster \\ Vienna University of Technology, Solid state physics, Wiedner Haupstr. 8-10, \\ A-1040 Vienna, Austria
}

\begin{abstract}
The switching times of submicron-sized magnets are in the order of pico- to nanoseconds. The switching speed is controlled by the geometric shape of the magnets, the intrinsic magnetic properties, and the orientation and strength of the applied field. Precessional motion governs the switching dynamics of small magnetic particles. If the rise time of the external field is faster than the relaxation of the magnetization towards the nearest local minimum, fast switching of the particles is possible at fields below the anisotropy field. The switching speed of thin film elements depends on the reversal mode. Two distinct reversal modes are found: (1) nucleation and expansion of reversed domains, and (2) nonuniform rotation of the magnetization. Domain nucleation occurs in randomly oriented, granular hcp-Co thin films and granular CoCrPt elements with perpendicular anisotropy. Rotational processes are found in granular fcc-Co thin films. The switching by rotation is about four times faster than switching by nucleation and expansion of domains. Switching by rotation can be enforced by applying a sufficiently large rotational field. Thermal activation decreases the switching time.
\end{abstract}

The switching dynamics of magnets has been an important issue in magnetic storage and sensor technology, since the development and application of magnetic core memories [1]. The detailed understanding of the switching process becomes important with increasing data rate in magnetic recording [2]. A high switching speed is desired in the recording layer of hard disks, in magnetic memory cells, and in magnetic sensor elements. A prominent example are magnetic random access memories (MRAMs) [3]. The switching process of a magnetic element sensitively depends on intrinsic properties, on the physical or chemical microstructure, and on the characteristics of the field pulse.

Numerical micromagnetics can provide a basic understanding of the switching dynamics [4]. Whereas small particles reverse by uniform rotation more complex magnetization processes are found if the size of the particle exceeds a critical length. Larger particles switch by the nucleation and expansion of reversed domains. Various intermediate switching modes may occur in mesoscopic elements. A smooth transition between the uniform rotation and domain nucleation is found as the particle size is increased.

This work reviews the basic physics of magnetization dynamics. Section 1 summarizes recent theoretical studies on the switching of magnetic elements. Sections 2 and 3 describe the equation of motion and precessional switching of 
small particles, respectively. Section 4 introduces the finite element method to treat magnetization processes in granular thin films. Section 5 presents finite element simulation of magnetization reversal in hcp-Co and fcc-Co thin film elements. The switching dynamics of perpendicular thin film elements for future discrete storage media are studied in section 6 . The effects of thermally activated processes on the switching speed is discussed in section 7. Section 8 summarizes the results.

\section{Introduction}

Experimentally, in situ domain observation using Lorentz electron microscopy [5] and time resolved magnetic imaging [6] provides a detailed understanding of domain formation and reversal processes. The numerical solution of the Gilbert equation of motion provides the theoretical background for the switching process of ferromagnetic structures. The switching time considerably depends on the Gilbert damping constant $\alpha$. Kikuchi [7] calculated the mimimum reversal time of a single domain sphere and a single domain thin film with in plane anisotropy. The critical value of the damping constant that minimizes the switching time is $\alpha=1$ for the sphere and $\alpha=0.01$ for the thin film. He and Doyle [8] solved the Landau-Lifshitz equation numerically, in order to investigate switching with very short field pulses. They conclude that switching times in the order of about 100 picoseconds are possible if the external field is applied at $90^{\circ}$ with respect to the anisotropy axis. If the rise time of the field pulse is less than a few nanoseconds and the Gilbert damping constant $\alpha<1$, switching can occur well below the Stoner-Wohlfarth limit.

Bauer and co-workers [9] investigated the switching properties of magnetic thin film elements subject to ultrashort, laterally localized magnetic field pulses numerically. The thin film is described by Stoner-like magnetic blocks. Field pulses of 4 ps duration cause magnetization reversal in both perpendicular and in-plane magnetized films. However, the time needed for the magnetization to reach equilibrium is about 550 ps. Mallinson [11] derived the switching time as a function of the field strength for fields parallel to the anisotropy direction. The switching time decreases with increasing external field.

Koch and coworkers [3] investigated the switching dynamics of micronsized magnetic thin films experimentally and numerically. They observed switching times well below 500 ps. Albuquerque and co-workers [12] presented a finite difference method to solve the Gilbert equation effectively for thin film structures as used in current-tunnel junction MRAM devices. A sequence of tailored field pulses causes a quasi-coherent switching in the subnanosecond regime, as short scale fluctuations are damped out quickly.

The coercive field of a magnet is a dynamic property. The measured coercivity significantly depends on the rate of change of the external field. Several experiments show an enhancement of the coercive field with decreasing pulse 
width of the external field [13]. The dynamic coercivity becomes important in ultra-high density and high data rate magnetic storage [14]. The gyromagnetic precession causes an increase of the coercive field at short times [15].

\section{The equation of motion}

The precessional motion of a magnetic moment in the absence of damping is described by the torque equation. According to quantum theory the angular momentum associated with a magnetic moment $\boldsymbol{m}$ is

$$
\boldsymbol{L}=\boldsymbol{m} / \gamma,
$$

where $\gamma$ is the gyromagnetic ratio. The torque on the magnetic moment, $\boldsymbol{m}$, exerted by an effective magnetic field, $\boldsymbol{H}_{\text {eff }}$, is

$$
\boldsymbol{T}=\boldsymbol{m} \times \boldsymbol{H}_{\mathrm{eff}} .
$$

The change of the angular momentum with time equals the torque,

$$
\frac{\partial}{\partial t}\left(\frac{\boldsymbol{m}}{\gamma}\right)=\boldsymbol{m} \times \boldsymbol{H}_{\mathrm{eff}},
$$

which describes the precession of the magnetic moment around the effective field. In equilibrium the change of the angular momentum with time is zero and thus the torque is zero. In order to describe the motion of the magnetic moment towards equilibrium, a viscous damping term can be included. A dissipative term proportional to the generalized velocity, $(\partial \boldsymbol{m} / \partial t)$, is added to the effective field. With $\eta$ being a positive constant, the dissipative term $-\eta(\partial \boldsymbol{m} / \partial t)$ slows down the motion of the magnetic moment and aligns $\boldsymbol{m}$ parallel to $\boldsymbol{H}_{\text {eff }}$. This gives the Gilbert equation of motion [16]

$$
\frac{\partial \boldsymbol{m}}{\partial t}=-|\gamma| \boldsymbol{m} \times\left(\boldsymbol{H}_{\mathrm{eff}}-\eta \frac{\partial \boldsymbol{m}}{\partial t}\right) .
$$

In a more convenient notation, the Gilbert equation writes

$$
\frac{\partial \boldsymbol{m}}{\partial t}=-|\gamma| \boldsymbol{m} \times \boldsymbol{H}_{\mathrm{eff}}+\frac{\alpha}{|\boldsymbol{m}|} \boldsymbol{m} \times \frac{\partial \boldsymbol{m}}{\partial t},
$$

where the dimensionless Gilbert damping constant $\alpha=|\gamma||\boldsymbol{m}| \eta$ was introduced. Multiplying (5) with $\boldsymbol{m}$. shows that the equation of motion conserves the norm of the magnetic moment, $|\boldsymbol{m}|$, since the right hand side vanishes:

$$
\boldsymbol{m} \cdot \frac{\partial \boldsymbol{m}}{\partial t}=\frac{1}{2} \frac{\partial(\boldsymbol{m} \cdot \boldsymbol{m})}{\partial t}=\frac{1}{2} \frac{\partial|\boldsymbol{m}|^{2}}{\partial t}=0 .
$$

Multiplying both sides of (5) with $\boldsymbol{m} \times$ gives

$$
\boldsymbol{m} \times \frac{\partial \boldsymbol{m}}{\partial t}=-|\gamma| \boldsymbol{m} \times\left(\boldsymbol{m} \times \boldsymbol{H}_{\mathrm{eff}}\right)+\frac{\alpha}{|\boldsymbol{m}|} \boldsymbol{m} \times\left(\boldsymbol{m} \times \frac{\partial \boldsymbol{m}}{\partial t}\right) .
$$


Using the vector identity

$$
\boldsymbol{a} \times(\boldsymbol{b} \times \boldsymbol{c})=(\boldsymbol{a} \cdot \boldsymbol{c}) \boldsymbol{b}-(\boldsymbol{a} \cdot \boldsymbol{b}) \boldsymbol{c}
$$

to rewrite the second term on the right hand side of $(7)$, we get

$$
\begin{aligned}
\boldsymbol{m} \times \frac{\partial \boldsymbol{m}}{\partial t}= & -|\gamma| \boldsymbol{m} \times\left(\boldsymbol{m} \times \boldsymbol{H}_{\mathrm{eff}}\right) \\
& +\frac{\alpha}{|\boldsymbol{m}|}\left(\boldsymbol{m} \cdot \frac{\partial \boldsymbol{m}}{\partial t}\right) \boldsymbol{m}-\frac{\alpha}{|\boldsymbol{m}|}(\boldsymbol{m} \cdot \boldsymbol{m}) \frac{\partial \boldsymbol{m}}{\partial t}, \\
\boldsymbol{m} \times \frac{\partial \boldsymbol{m}}{\partial t}= & -|\gamma| \boldsymbol{m} \times\left(\boldsymbol{m} \times \boldsymbol{H}_{\mathrm{eff}}\right)-\alpha|\boldsymbol{m}| \frac{\partial \boldsymbol{m}}{\partial t} .
\end{aligned}
$$

If we substitute this result into (5), we obtain the Landau-Lifshitz-Gilbert (LLG) equation $[17,10]$

$$
\frac{\partial \boldsymbol{m}}{\partial t}=-\frac{|\gamma|}{1+\alpha^{2}} \boldsymbol{m} \times \boldsymbol{H}_{\mathrm{eff}}-\frac{|\gamma| \alpha}{\left(1+\alpha^{2}\right)|\boldsymbol{m}|} \boldsymbol{m} \times\left(\boldsymbol{m} \times \boldsymbol{H}_{\mathrm{eff}}\right) .
$$

The first term on the right hand side of (10) describes the gyromagnetic precession, the second term describes the damping. Fig. 1 illustrates the motion of the magnetic moment, $\boldsymbol{m}$, under the influence of the effective field, $\boldsymbol{H}_{\mathrm{eff}}$.

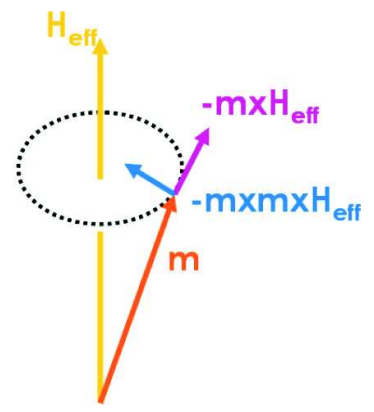

Fig. 1. Damped gyromagnetic precession of a single magnetic moment, $\boldsymbol{m}$, towards the effective magnetic field $\boldsymbol{H}_{\text {eff }}$ according to the Landau-Lifshitz Gilbert equation (10).

Equation (10) may be used to study the switching dynamics of small magnetic particles. If the particles are sufficiently small the magnetization reverses by quasi-uniform rotation [18]. Then the magnetization remains uniform during the reversal process and the only contributions to the effective field are the anisotropy field, the demagnetizing field, and the external field:

$$
\begin{aligned}
\boldsymbol{H}_{\mathrm{eff}} & =\left\{\frac{2 K_{\mathrm{u}}}{J_{\mathrm{s}}}+\frac{J_{\mathrm{s}}}{\mu_{0}}\left(N_{\perp}-N_{\|}\right)\right\} \frac{(\boldsymbol{m} \cdot \boldsymbol{u})}{|\boldsymbol{m}|} \boldsymbol{u}+\boldsymbol{H}_{\mathrm{ext}}, \\
& =\frac{2 K}{J_{\mathrm{s}}} \frac{(\boldsymbol{m} \cdot \boldsymbol{u})}{|\boldsymbol{m}|} \boldsymbol{u}+\boldsymbol{H}_{\mathrm{ext}}
\end{aligned}
$$


Here $K_{\mathrm{u}}$ is the uniaxial magnetocrystalline anisotropy constant and $J_{\mathrm{s}}$ is the magnetic polarization. If $V$ is the particle volume the total magnetic moment of the particle is $|\boldsymbol{m}|=V J_{\mathrm{s}} / \mu_{0} . N_{\|}$and $N_{\perp}$ are the demagnetizing factors parallel and normal to the symmetry axis, which is assumed to be parallel to the magnetocrystalline anisotropy direction $\boldsymbol{u}$. In (12) the effective anisotropy constant

$$
K=K_{\mathrm{u}}+\frac{J_{\mathrm{s}}^{2}}{2 \mu_{0}}\left(N_{\perp}-N_{\|}\right)
$$

has been introduced.

Equations (10) and (12) describes the switching process of a small magnetic particle under the influence of an external field.

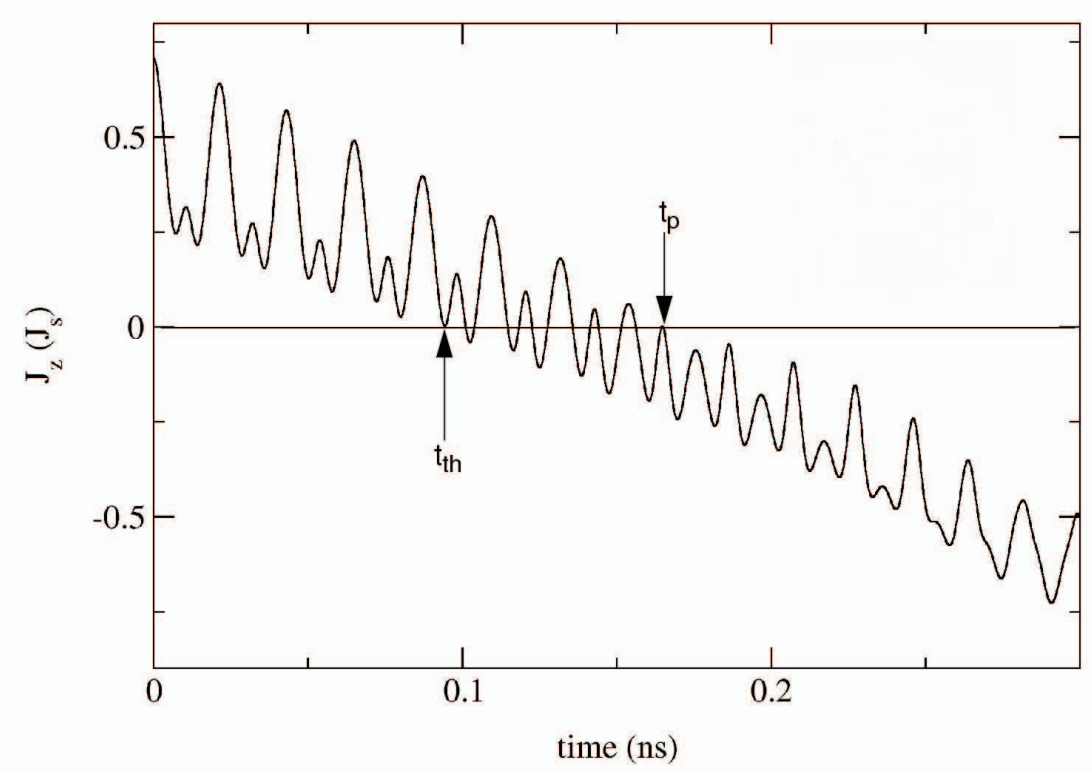

Fig. 2. The average magnetization parallel to the long axis of a small magnetic particle as a function of time for an angle $\beta=1^{\circ}$ between the external field and the anisotropy direction. The theoretical switching time $t_{t h}$ and the practical switching time $t_{p}$ are marked.

\section{Small magnetic particles}

A theoretical understanding of high speed switching of magnetic grains becomes increasingly important as the data rate of magnetic recording media approaches the $\mathrm{GHz}$ regime. At such high frequencies gyromagnetic effects 


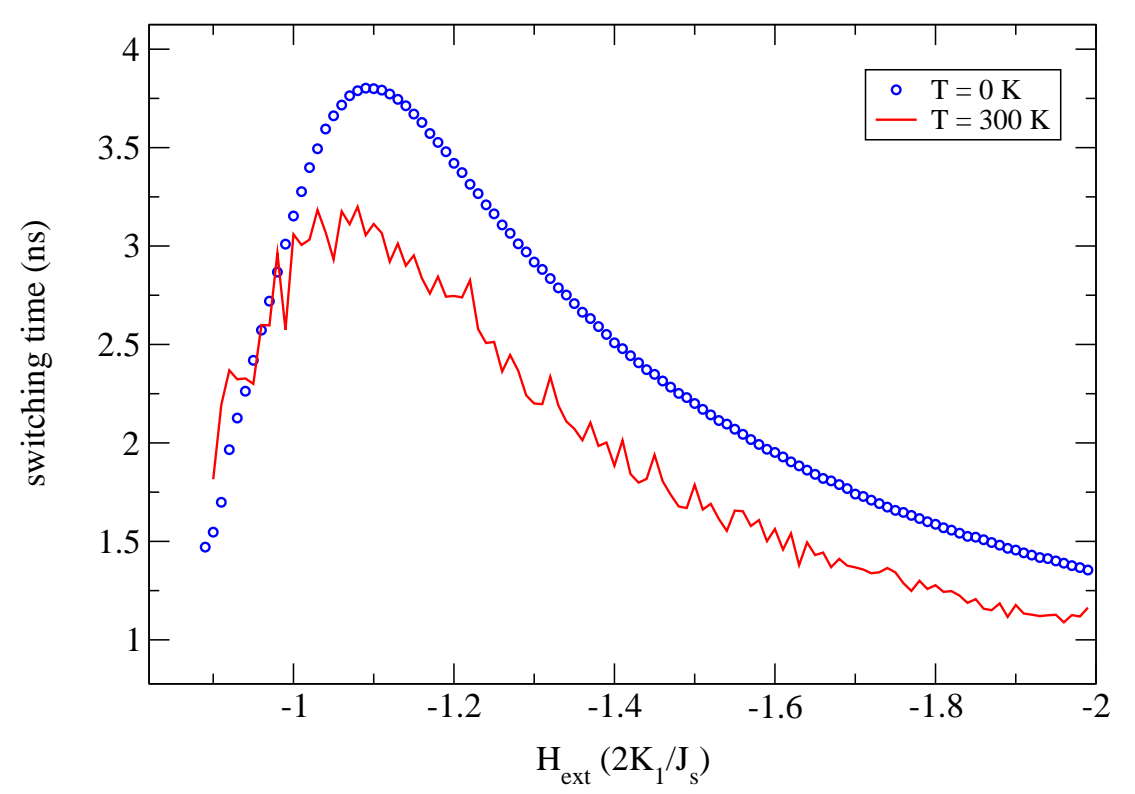

Fig. 3. Switching time of a small magnetic particle as a function of the field strength. Circles: $\beta=1^{\circ}, \mathrm{T}=0 \mathrm{~K}$, solid line: $\beta=1^{\circ}, \mathrm{T}=300 \mathrm{~K}$.

We consider a small particle with the following intrinsic magnetic properties: $J_{\mathrm{s}}=0.5 \mathrm{~T}$, and $K=3 \times 10^{5} \mathrm{~J} / \mathrm{m}^{3}$. The Gilbert damping constant is $\alpha=0.02$. The easy axis is parallel to the $z$-axis. Initially the particle is magnetized parallel to the positive $z$-direction. An external field is applied instantaneously at an angle $\beta$ between the field and the negative $z$-axis. We define the theoretical switching time as the minimum length of a field pulse which is required to switch the particle. For shorter field pulses the magnetization may precess back and no switching occurs. A suitable definition of a practical switching time is the critical duration of the field pulse, $t_{\mathrm{p}}$, so that all field pulses longer than $t_{\mathrm{p}}$ will switch the particle. Fig. 2 shows the time evolution of the average magnetic polarization parallel to the $z$-axis, $J_{\mathrm{z}}=\mu_{0}\langle\boldsymbol{m} \cdot \hat{\boldsymbol{z}}\rangle / V$. The magnetization oscillates owing to gyromagnetic precession. This effect is more pronounced with larger angle $\beta$. The arrows in Fig. 2 show the theoretical switching time $t_{\text {th }}$ and the practical switching time $t_{\mathrm{p}}$, where $t_{\mathrm{th}}$ is the first intersection of the $J_{z}(t)$ curve with the abscissa and $t_{\mathrm{p}}$ is the last intersection of the $J_{z}(t)$ curve with the abscissa. A field pulse of the duration $t_{\text {th }}$ may not switch the particle, whereas a pulse with a duration greater than $t_{\mathrm{p}}$ guarantees switching. Fig. 3 shows the switching time 
$t_{\mathrm{n}}$ as a function of the strength of the external field. It is conspicuous that

A
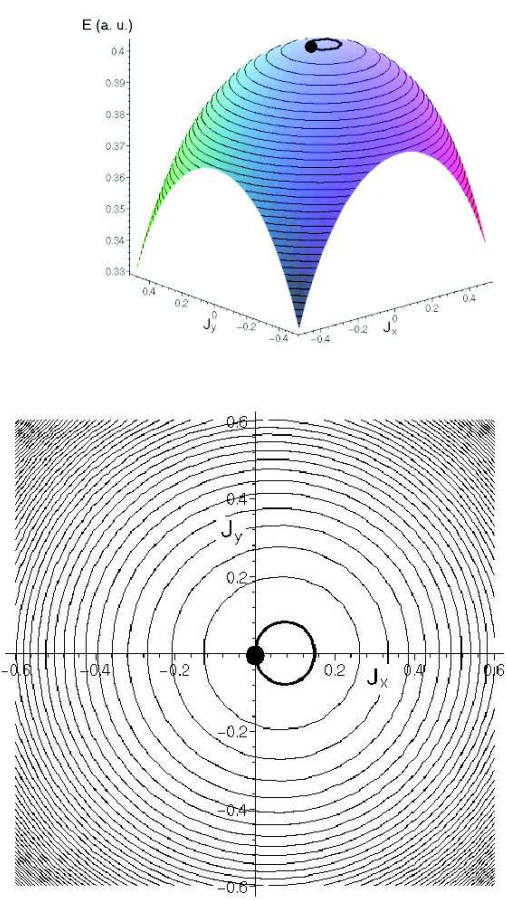

B
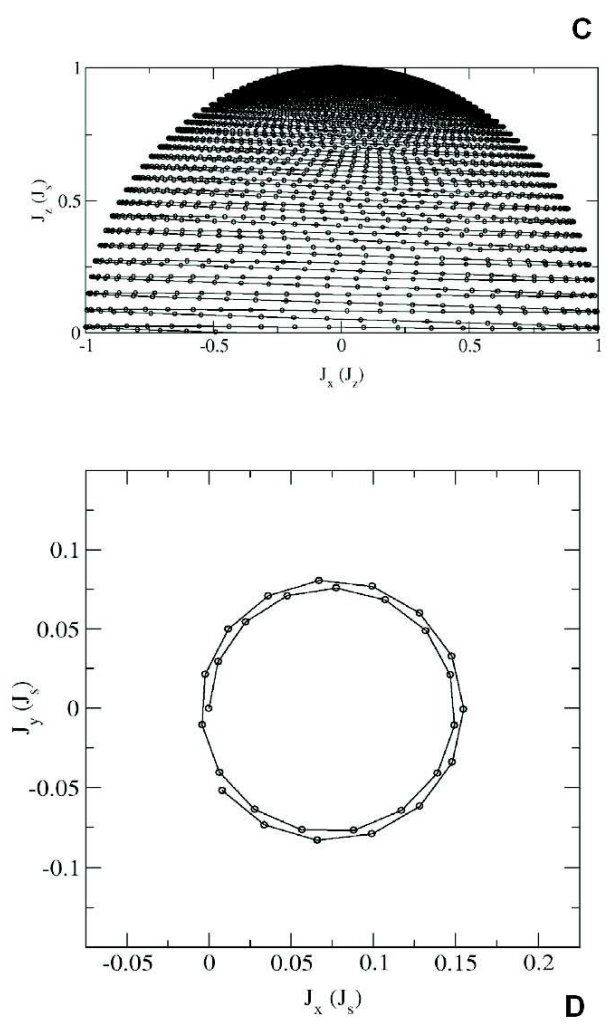

Fig. 4. Energy landscape and paths of the magnetic polarization towards the switched state when an external field of $H_{\text {ext }}=-1.3\left(2 K / J_{\mathrm{s}}\right)$ is applied. (A) and (B) show the energy landscape and the contour plot in terms of the normalized magnetic polarization, respectively. (C) and (D): Locus of the polarization as a function of time. The circles are plotted every 0.005 ns. (D) shows only the first $0.15 \mathrm{~ns}$ after the application of the external field.

The path the system follows through the energy landscape may explain the switching behavior more clearly. Figs. 4 and 5 show the path of the magnetization through the energy landscape and the locus of the magnetization just after the application of the field $H_{\text {ext }}=-0.9\left(2 K / J_{\mathrm{s}}\right)$ and $H_{\text {ext }}=-1.3\left(2 K / J_{\mathrm{s}}\right)$, respectively. The energy landscape is plotted as a function of $J_{x} / J_{\mathrm{s}}$ and $J_{y} / J_{\mathrm{s}}$. Before the application of the external field, 

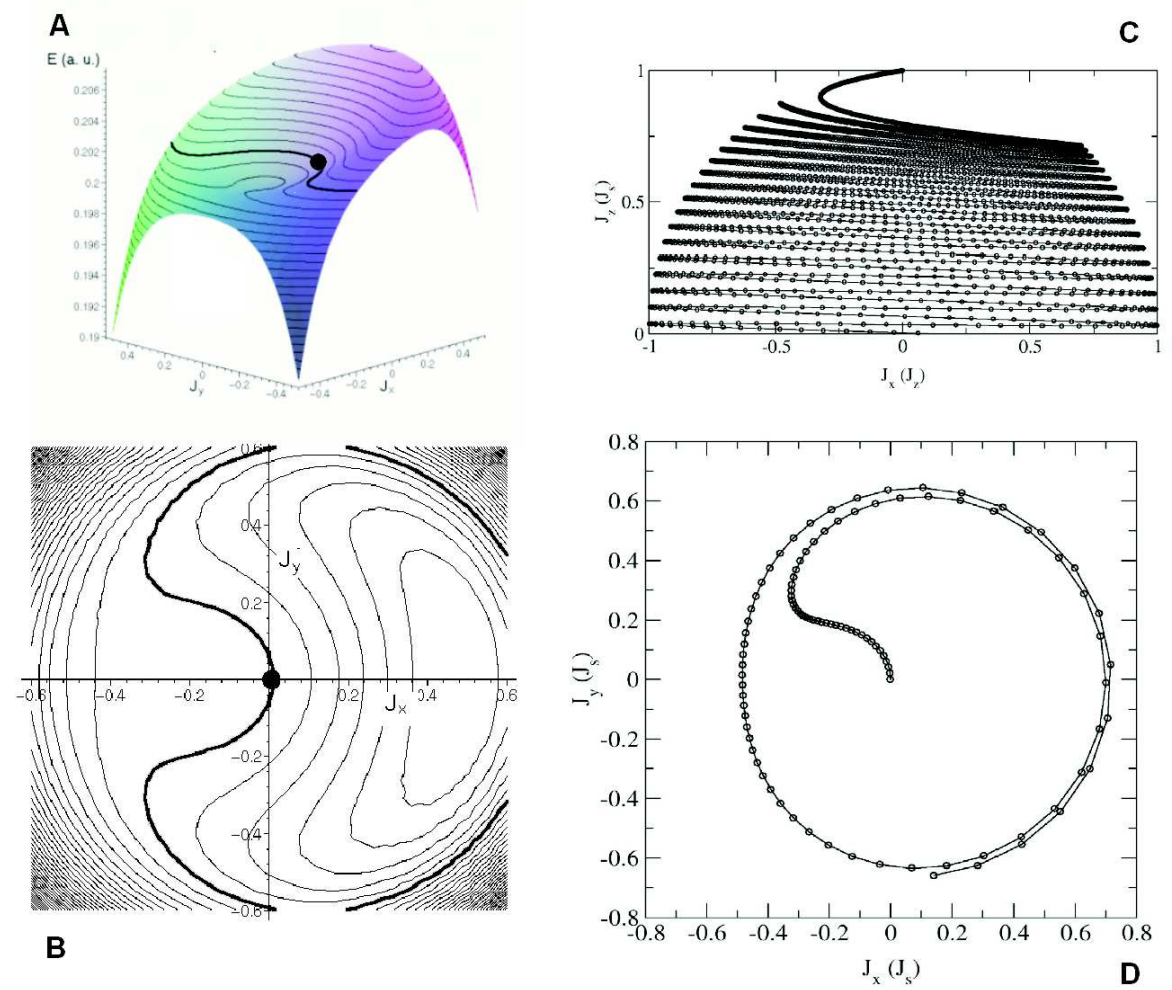

Fig. 5. Energy landscape and paths of the magnetic polarization towards the switched state when an external field of $H_{\text {ext }}=-0.9\left(2 K / J_{\mathrm{s}}\right)$ is applied. (A) and (B) show the energy landscape and the contour plot in terms of the normalized magnetic polarization, respectively. (C) and (D): Locus of the polarization as a function of time. The circles are plotted every $0.005 \mathrm{~ns}$. (D) shows only the first 0.5 ns after the application of the external field.

the system is in equilibrium at $J_{x}=J_{y}=0$ marked with a dark dot in (A) and (B) in Figs. 4 and 5.

If a field of $H_{\text {ext }}=-1.3\left(2 K / J_{\mathrm{s}}\right)$ is applied the energy landscape suddenly changes. The system is no longer in equilibrium. A well defined maximum is formed due to the Zeeman energy which contributes most to the total energy. The initial state is close to the maximum. Due to the precession term in the LLG equation, the magnetization moves along a path with almost constant energy as shown in Fig. 4 (D). If the damping constant is small the magnetization will initially follow this path, indicated by the bold line in Fig. 4 (A and B). During this motion the angle between the magnetization and the effective field remains small and it precesses several times around the effective field, with $J_{z}$ almost 1 (Fig. $4 \mathrm{C}$ ). Since for $J_{z}$ almost 1 the torque $\boldsymbol{m} \times \boldsymbol{H}$ is small, the relaxation towards the reversed state is slow. A large 
switching time occurs. Only for fields exceeding 1.1 times the anisotropy field, the switching time decreases. This is due to the linear increase of the torque with the strength of the effective field.

If the external field is comparable with the anisotropy field a more complex energy landscape is obtained, because anisotropy energy and Zeeman energy contribute in the same order of magnitude to the total energy. The bold line in the contour plot in Fig. 5 (B) gives the path of the magnetization for an external field of $H_{\text {ext }}=-0.9\left(2 K / J_{\mathrm{s}}\right)$. The precession drives the magnetization away from the effective field (Fig. 5 D) which leads to a high torque. Fast switching occurs when the magnetization does not relax back to the local minimum shown in Fig. 5 (A) but moves towards the local minimum which corresponds to the reversed state. For an external field $\left|H_{\text {ext }}\right| \geq 0.89\left(2 \mathrm{~K} / J_{\mathrm{s}}\right)$ the initial motion of the magnetization along a path with nearly constant energy leads to magnetization reversal. The minimum switching time was $1.4 \mathrm{~ns}$ for $\left|H_{\text {ext }}\right|=0.89\left(2 \mathrm{~K} / J_{\mathrm{s}}\right)$, whereas a field of $\left|H_{\text {ext }}\right|>1.9\left(2 \mathrm{~K} / J_{\mathrm{s}}\right)$ is required to switch the particle within the same time for large external fields.

The above results show that fast switching of small particles is possible in external fields below the anisotropy field. Switching may occur well below the Stoner-Wohlfarth limit [19]. Investigating the switching dynamics of thin films, Fassbinder [20] showed that it is possible to tailor the magnetic precession, which in turn can be used to optimize the performance of magnetic devices. Both numerical simulations and time-resolved magneto-optic Kerr magnetometry demonstrate that the trajectory of the magnetization upon application of a field pulse depends on the effective field, the damping parameter, and the magnetic field pulse parameters.

\section{Finite element micromagnetics}

Magnetic thin film elements as used for magnetic sensors or MRAM cells are composed of small magnetic grains. The individual grains of a magnetic nanoelement interact by exchange and magnetostatic interactions. Micromagnetics combines Maxwell's equations for the magnetostatic field with the equation of motion describing the time evolution of the magnetization. The local arrangement of the magnetic moments follows from the complex interaction between intrinsic magnetic properties such as the magnetocrystalline anisotropy and the physical/chemical microstructure of the material. The numerical integration of the partial differential equations which describe the dynamic response of a magnetic system under the influence of an external field provides a detailed understanding of the microscopic processes that determine the macroscopic magnetic properties like switching time and switching field.

In granular films the magnetocrystalline anisotropy constant and the magnetocrystalline anistropy direction may vary from grain to grain. The demagnetizing field is non-uniform, owing to sharp edges and corners. As a conse- 
quence the magnetization reversal process is non-uniform. The exchange field becomes important, in addition to the contributions to the effective field given in (12). The effective field follows Gibbs' free energy [21]

$$
E=\int d V\left\{\frac{A}{J_{\mathrm{s}}^{2}} \sum_{i=1}^{3}\left(\nabla J_{i}\right)^{2}+f_{\mathrm{ani}}(\boldsymbol{J})-\frac{1}{2} \boldsymbol{J} \cdot \boldsymbol{H}_{\mathrm{d}}-\boldsymbol{J} \cdot \boldsymbol{H}_{\mathrm{ext}}\right\}
$$

The total energy of complex microstructures can be evaluated using the finite element method. The magnetic polarization, $\boldsymbol{J}$, is interpolated with piecewise linear functions on a tetrahedral finite element mesh. $f_{\text {ani }}$ is the magnetocrystalline anisotropy energy density. The LLG equation (10) is assumed to hold at every node of the finite element mesh. Using spatial averaging we assign a magnetic moment

$$
\boldsymbol{m}^{(i)}=\frac{1}{\mu_{0}} \int_{V_{i}} \boldsymbol{J}(\boldsymbol{r}) \mathrm{d}^{3} r
$$

to node $i$ of the finite element mesh. The box volumes $V_{i}$ have the following properties

$$
\begin{aligned}
\sum_{i=1}^{N} V_{i} & =\int \mathrm{d}^{3} r, \\
V_{i} \cap V_{j} & =0 \quad \text { for } \quad i \neq j,
\end{aligned}
$$

where the sum in (16) runs from 1 to the total number of nodes of the finite element mesh, $N$. The effective field at node $i$ can be approximated as

$$
\boldsymbol{H}_{\mathrm{eff}}^{(i)}=-\frac{1}{\mu_{0}} \frac{\partial E}{\partial \boldsymbol{m}^{(i)}} .
$$

The finite element method is a highly flexible tool to describe magnetization processes, since it is possible to incorporate the physical grain structure and to adjust the finite element mesh according to the local magnetization [22]. The space discretization of the LLG equation using (15) to (18) leads to a system of $3 N$ ordinary differential equations. It is solved using a backward differentiation method [23].

In equation (14) $\boldsymbol{H}_{\mathrm{d}}$ is the demagnetizing field. It arises from magnetic volume charges, $\nabla \cdot \boldsymbol{J} / \mu_{0}$, within the magnetic particles and magnetic surface charges, $\boldsymbol{J} \cdot \boldsymbol{n} / \mu_{0}$ at the boundaries. The demagnetizing field can be computed from a magnetic scalar potential $\boldsymbol{H}_{\mathrm{d}}=-\nabla U$ which satisfies the following boundary value problem

$$
\begin{array}{ll}
\nabla^{2} U(\boldsymbol{r})=\nabla \cdot \boldsymbol{J}(\boldsymbol{r}) / \mu_{0} & \text { for } \boldsymbol{r} \in \Omega_{\mathrm{int}}, \\
\nabla^{2} U(\boldsymbol{r})=0 & \text { for } \boldsymbol{r} \in \Omega_{\mathrm{ext}},
\end{array}
$$


where $\Omega_{\text {int }}$ and $\Omega_{\text {ext }}$ denote the space within and outside the magnet, respectively. At the boundary $\Gamma$ the boundary conditions

$$
U^{\text {int }}=U^{\text {ext }}, \quad\left(\nabla U^{\text {int }}-\nabla U^{\text {ext }}\right) \cdot \boldsymbol{n}=(\boldsymbol{J} \cdot \boldsymbol{n}) / \mu_{0}
$$

hold. Here $\boldsymbol{n}$ denotes the outward pointing normal unit vector on $\Gamma$. The magnetic scalar potential is regular at infinity

$$
U \propto 1 / \boldsymbol{r} \text { for } \boldsymbol{r} \rightarrow \infty
$$

In order to solve equations (19) to (22), we apply a hybrid finite element / boundary element method [24].

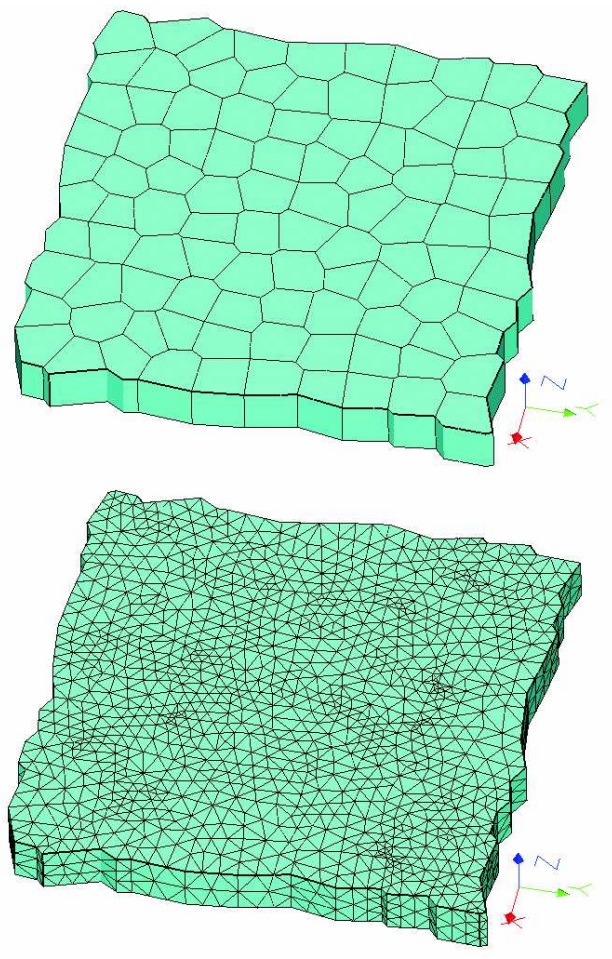

Fig. 6. Schematic granular structure of a square thin film element of $100 \times 100 \times 10 \mathrm{~nm}^{3}$ consisting of 100 grains with a grain size of about $10 \mathrm{~nm}$. Discretization into tetrahedral finite elements used for the numerical simulation of the switching behavior of randomly oriented Co grains.

\section{Magnetization reversal in thin film Co elements}

Magnetic switching of mesoscopic and nanostructured thin film elements, nanowires and particles becomes increasingly important in magnetic storage, microsensors and magneto electronic devices. Nanostructured magnetic elements may be used as storage elements [25], field sensors [26], or logic gates 
[27]. In this section, three dimensional (3D) micromagnetic simulations of the reversal process within granular nanoscale squares are performed to examine the influence of magnetocrystalline anisotropy on the switching behavior of thin film elements consisting of randomly oriented uniaxial or cubic Co grains. Reference calculations show how the switching process induced by a rotating field differs from the magnetization reversal of granular thin film elements under the influence of a constant unidirectional field.

Russek and coworkers [28] investigated the switching dynamics of high speed magnetic devices. They compare experimental data and micromagnetic simulations and present a detailed picture of rotational dynamics in magnetic thin film elements.

\subsection{Polycrystalline Co elements}

The basic geometry of the granular thin film element is shown in Fig. 6. The thin, nanostructured square element with dimensions of $100 \times 100 \times 10 \mathrm{~nm}^{3}$ consists of 100 irregularly shaped grains with an average diameter of about $10 \mathrm{~nm}$. The grains have uniaxial magnetocrystalline anisotropy and are 3D randomly oriented. For the simulations the intrinsic bulk properties of hcpCo $\left(J_{\mathrm{s}}=1.76 \mathrm{~T}, K_{1}=0.45 \mathrm{MJ} / \mathrm{m}^{3}, K_{2}=0.15 \mathrm{MJ} / \mathrm{m}^{3}\right.$, and $A=13 \mathrm{pJ} / \mathrm{m}$ $[29])$ or fcc-Co $\left(J_{\mathrm{s}}=1.76 \mathrm{~T}, K_{1}=0.05 \mathrm{MJ} / \mathrm{m}^{3}, K_{2}=0\right.$, and $A=13 \mathrm{pJ} / \mathrm{m}$ $[30])$ were used.

\subsection{1 hep-Co films}

To study the switching dynamics an external field is applied inplane parallel to an edge. The field is varied linearly in time with different speeds (sweep rate). Fig. 7 shows the calculated dynamic coercivity of the hcp-Co element.

$H_{\mathrm{c}}$ depends on both the damping constant $\alpha$ and the sweep rate of the external field. $\left|H_{\mathrm{c}}\right|$ increases with increasing sweep rate of the external field. This effect itself also depends on $\alpha$, and is stronger for higher damping constants. For weak damping, $\alpha \leq 0.1$, the coercive field increases linearly with the sweep rate. For high damping the slope of the $H_{\mathrm{c}}\left(\mathrm{d} H_{\text {ext }} / \mathrm{d} t\right)$ curve decreases at high sweep rates.

Figs. 8 and 9 give the time evolution of the magnetization component parallel to the direction of the applied field for a sweep rate of $-28 \mathrm{kA} /(\mathrm{m} \cdot \mathrm{ns})$ and $-2800 \mathrm{kA} /(\mathrm{m} \cdot \mathrm{ns})$, respectively. Increasing the sweep rate by two orders of magnitude reduces the switching time from about 5 ns to 0.12 ns. Fig. 8 shows that it is possible to switch the Co element with an opposing field of $120 \mathrm{kA} / \mathrm{m}$. This field value is reached after $0.05 \mathrm{~ns}$ in the fast switching experiment shown in Fig. 8. The rapid increase of the opposing field leads to a monotonic decrease of $\left\langle J_{y}\right\rangle / J_{\mathrm{s}}$ with time.

The micromagnetic simulations show that for the Co thin films with uniaxial anisotropy (hcp-Co) the magnetization reversal mechanisms are similar 


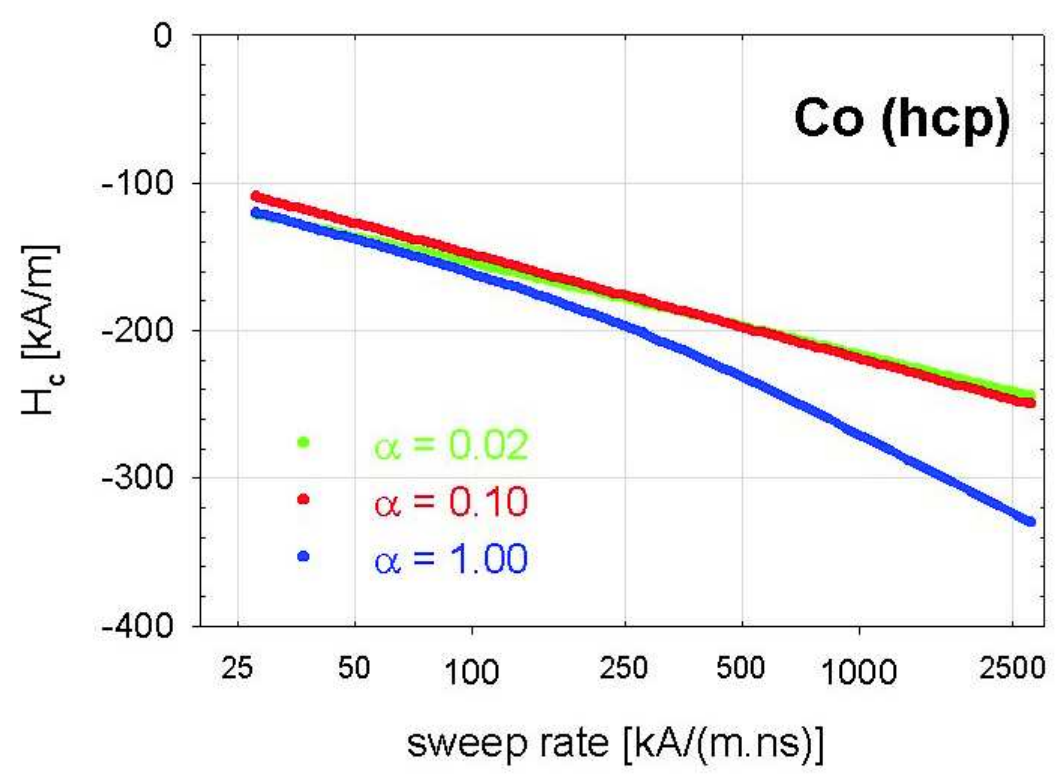

Fig. 7. Polycrystalline hcp-Co. Dynamic coercivity as a function of the field sweep rate for three different values of the damping constant $\alpha$.

for low and high damping parameters and low and high sweep rates. Fig. 10 illustrates the switching process of polycrystalline hcp-Co elements. Magnetization reversal starts by the formation of end domains where the magnetization rotates into a direction perpendicular to the applied field. This process reduces both the Zeeman energy and the magnetostatic energy. Magnetic surface charges are suppressed, since the magnetization is nearly parallel to the edges (Fig. 10a). As the external field is increased the magnetization reverses close to the edges that are parallel to the external field (Fig. 10b). These reversed domains expand along the edges while the magnetization remains in its original direction in the core of the element (Fig. 10c). Finally, the magnetization in the center starts to rotate into the field direction (Fig. 10d). The transient states show that the magnetization reversal in hcp-Co films occurs by the nucleation and expansion of reversed domains. The switching time for the process shown in Fig. 10 is 0.72 ns.

\subsection{2 fcc-Co films}

The magnetocrystalline anisotropy strongly influences the switching properties of the granular Co thin film element. The numerical simulations show a decrease of the switching time and field in the case of randomly oriented grains with cubic anisotropy (fcc-Co). Again the external field is applied inplane parallel to an edge and varied linearly in time with different speeds. 


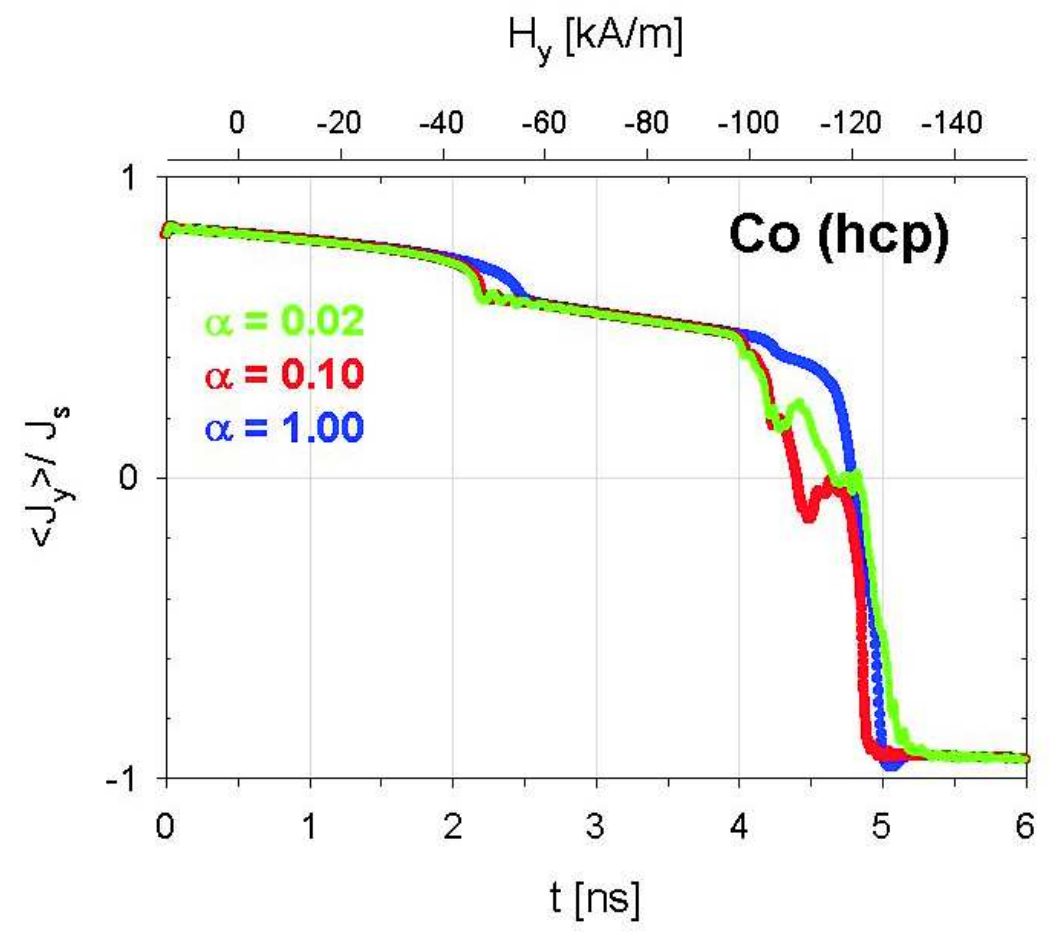

Fig. 8. Slow switching of polycrystalline hcp-Co. Time evolution of the magnetization component parallel to the direction of the applied field for three different values of the damping constant $\alpha$. The sweep rate of the applied field is $-28 \mathrm{kA} /(\mathrm{m} \cdot \mathrm{ns})$.

Fig. 11 shows the calculated dynamic coercivity of the fcc-Co element. Owing to the weak magnetocrystalline anisotropy both the coercivity and the switching time decreases in the fcc-Co films as compared to the hcp-Co films. The dynamic coercivity as a function of the sweep rate shows two different regimes. For low sweep rates the dynamic coercivity is less than $40 \mathrm{kA} / \mathrm{m}$ which is about three times smaller than the dynamic coercivity of hcp-Co. For high sweep rates the difference in the dynamic coercivity between hcpand fcc-Co films is less pronounced.

Figs. 12 and 13 compare the time evolution of the magnetization component parallel to the direction of the applied field for different sweep rates. The switching time is less than $2 \mathrm{~ns}$ for a sweep rate of $-28 \mathrm{kA} /(\mathrm{m} \cdot \mathrm{ns})$ and less than $0.1 \mathrm{~ns}$ for $-2800 \mathrm{kA} /(\mathrm{m} \cdot \mathrm{ns})$.

Whereas the magnetization becomes strongly inhomogeneous during the switching of the hcp-Co film, the magnetization rotates rather uniformly during the reversal of the fcc-Co film. Fig. 14 shows that both the magnetization near the edges and the magnetization in the center of the film rotate simultaneously. Again end domains are formed which have a magnetization 


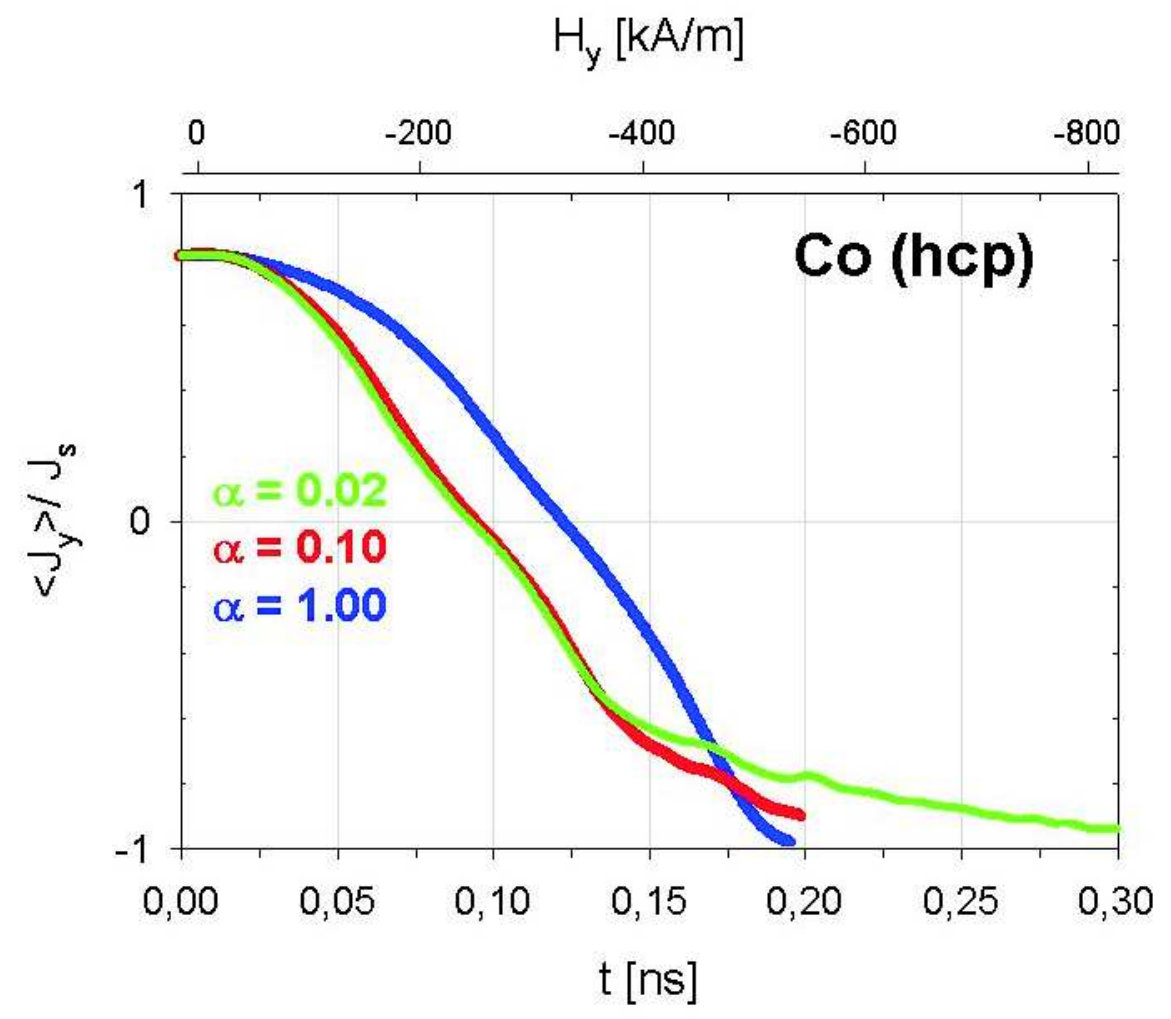

Fig. 9. Fast switching of polycrystalline hcp-Co. Time evolution of the magnetization component parallel to the direction of the applied field for three different values of the damping constant $\alpha$. The sweep rate of the applied field is $-2800 \mathrm{kA} /(\mathrm{m} \cdot \mathrm{ns})$.

perpendicular to the direction of the applied field (Fig. 14a). As the external field is increased the magnetization in the center rotates parallel to the magnetization in the end domains (Fig. 14b). Then the magnetization reverses in the center followed by the reversal of the magnetization near the edges that are parallel to the field direction (Fig. 14c and Fig. 14d). The switching time for this process is $0.16 \mathrm{~ns}$.

\subsection{Switching in a rotational field}

The comparison of the magnetization reversal process between hcp- and fccCo films shows that the switching time of fcc-Co films is about a factor of $1 / 4$ smaller than the switching time of hcp-Co films. The higher switching speed of fcc-Co might be attributed to the more uniform magnetization reversal process in fcc-Co as compared to hcp-Co. In uniaxial Co switching occurs by the nucleation and expansion of reversed domains leading to strongly inhomoge- 
(a)

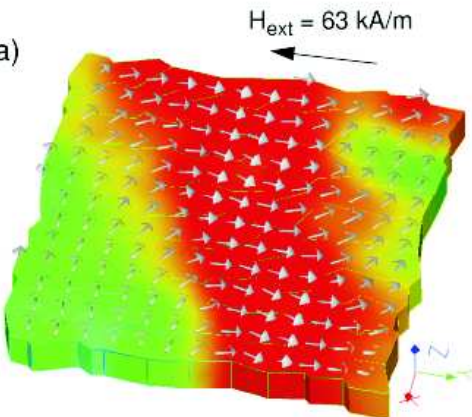

(c)

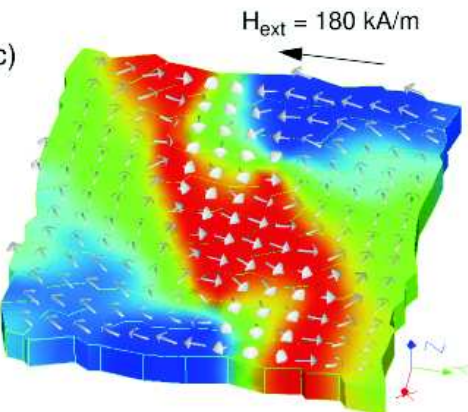

(b)
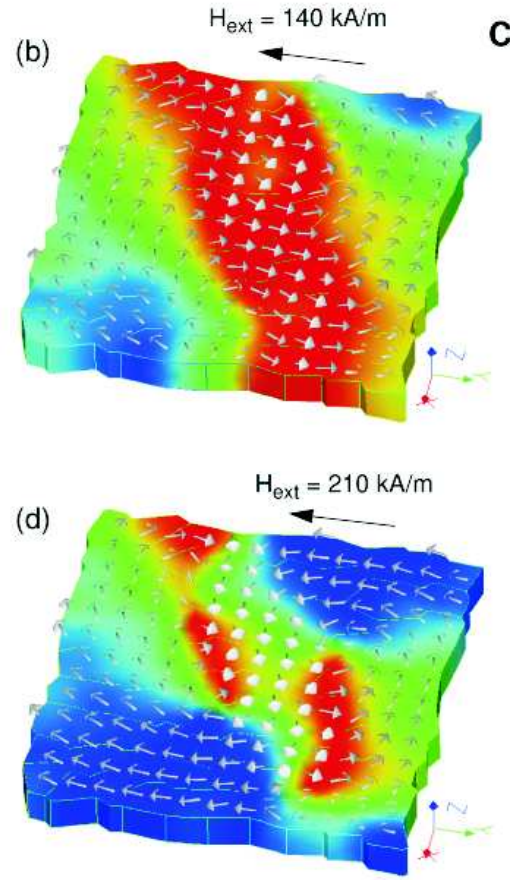

Fig. 10. Magnetization distribution of polycrystalline hcp-Co during the application of a unidirectional field with a sweep rate of $-280 \mathrm{kA} /(\mathrm{m} \cdot \mathrm{ns})$. The Gilbert damping constant is $\alpha=0.1$. The grey scale maps the magnetization component parallel to the field direction. (a) $\left\langle J_{y}\right\rangle / J_{\mathrm{s}}=0.6$, (b) $\left\langle J_{y}\right\rangle / J_{\mathrm{s}}=0.3$, (c) $\left\langle J_{y}\right\rangle / J_{\mathrm{s}}=0$, and $(\mathrm{d})\left\langle J_{y}\right\rangle / J_{\mathrm{s}}=-0.3$.

neous transient magnetization configurations. In cubic Co the magnetization rotates nonuniformly.

In order to investigate the effects of the reversal mode on the switching speed of thin film elements we compare the switching process under the influence of a unidirectional and a rotational external field. Fig. 15 compares the time evolution of the magnetization during the application of a unidirectional field and a rotating field. The results clearly show that the switching speed under the influence of a rotating field is more than four times faster than the switching speed in a constant reversed field.

Under the influence of a constant rotating field the magnetization tries to follow the external field direction. At low fields $\left(\left|H_{\text {ext }}\right|=140 \mathrm{kA} / \mathrm{m}\right)$ the magnetization starts to rotate near the flat ends of the square to reduce the magnetostatic energy, followed by the reversal of the center. The magnetization reversal process resembles the process shown in Fig. 14. At high fields $\left|H_{\text {ext }}\right|=280 \mathrm{kA} / \mathrm{m}$ the magnetization follows the field direction in each grain.

Fig. 16 shows the difference in the reversal modes which are obtained in a constant unidirectional field and in a rotating field. In a constant uni- 


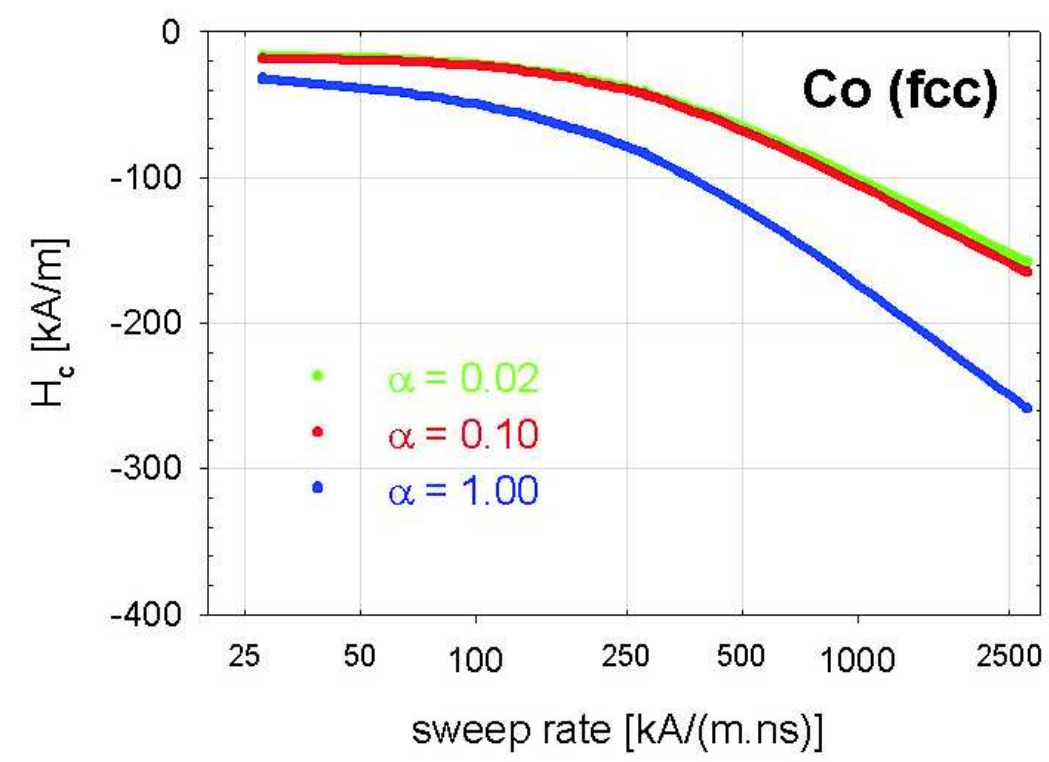

Fig. 11. Polycrystalline fcc-Co. Dynamic coercivity as a function of the field sweep rate for three different values of the damping constant $\alpha$.

directional field magnetization reversal starts by the nucleation of reversed domains at opposite corners. The reversed domains expand and cause the reversal of the entire film. In a rotating field the magnetization remains almost uniform during reversal as the magnetization tries to follow the field direction.

\section{Switching of perpendicular discrete media}

Discrete media show great potential for future ultra-high density magnetic recording. Discrete media consist of arrays of discrete, for example ion-beam patterned magnetic elements [31], each of which can store one bit of data. Ideally, the storage density is then equal to the surface density of the elements. In patterned media, each discrete element is exchange isolated from other elements, but inside each element polycrystalline grains are strongly exchange-coupled, behaving more like a larger single magnetic grain. Nevertheless the micromagnetic simulations show that a single island reverses incoherently by the expansion of a small reversed nucleus.

Beside the effort of reaching higher area densities, another crucial point for magnetic recording applications is a fast writing speed in order to guarantee high data rates. We studied the reversal dynamics of a single $\mathrm{CoCrPt}$ island with perpendicular anisotropy. The thin film element has a thickness of $21 \mathrm{~nm}$ and consists of 49 columnar grains. The average grain diameter is $10 \mathrm{~nm}$. The 


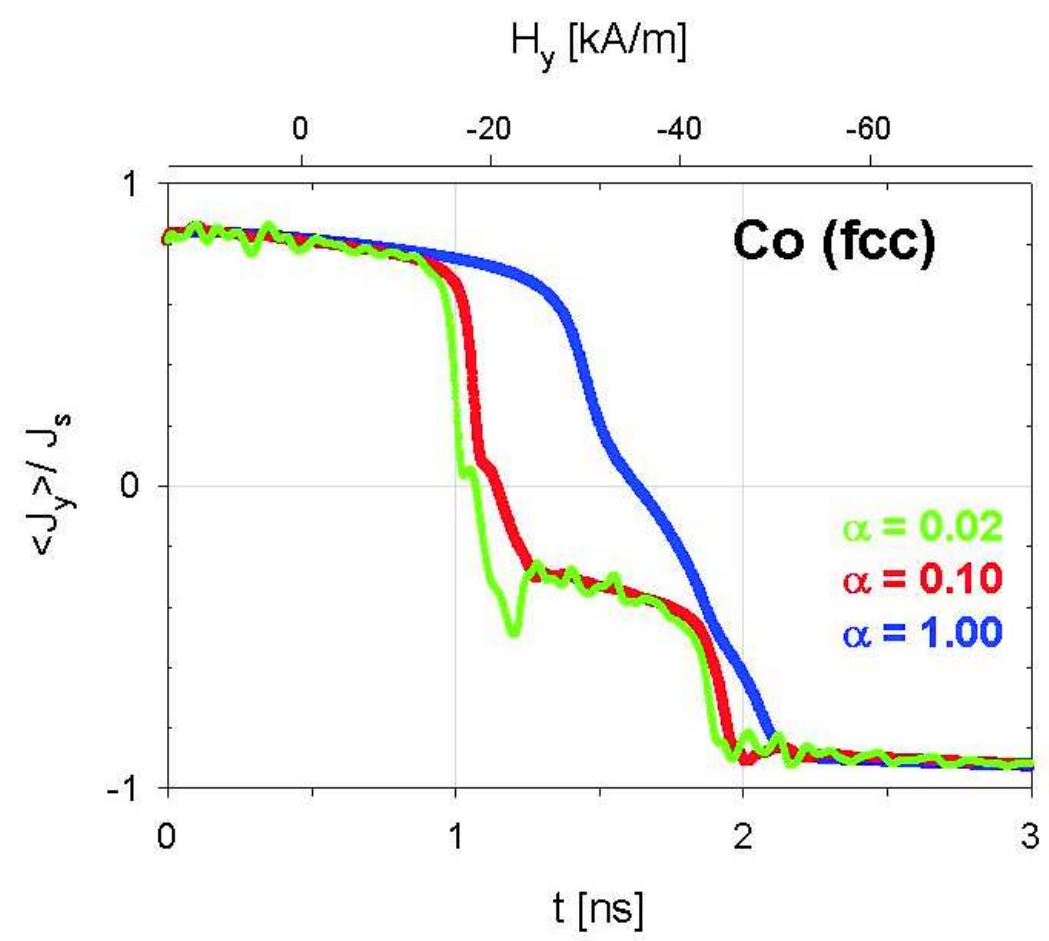

Fig. 12. Slow switching of polycrystalline fcc-Co. Time evolution of the magnetization component parallel to the direction of the applied field for three different values of the damping constant $\alpha$. The sweep rate of the applied field is $-28 \mathrm{kA} /(\mathrm{m} \cdot \mathrm{ns})$.

material parameters are assumed as follows: $K_{\mathrm{u}}=0.3 \mathrm{MJ} / \mathrm{m}^{3}, J_{\mathrm{s}}=0.5 \mathrm{~T}$, and $A=10 \mathrm{pJ} / \mathrm{m}$. The magnetocrystalline anisotropy axis is perpendicular to the film plane with a random deviation from the film normal up to $10^{\circ}$.

Fig. 17 shows the dynamic coercivity calculated for a single CoCrPt island. The simulations were made for low damping $(\alpha=0.02)$ and for high damping $(\alpha=1)$. The dynamic coercivity depends both on the damping constant and on the sweep rate of the external field. The coercivity increases with increasing sweep rate of the external field. Thus a higher field has to be applied for higher writing speeds. This effect itself also depends on $\alpha$, and is stronger for smaller damping constants.

Figs. 18 and 19 show that the magnetization reversal starts at an external field of $H_{\text {ext }}=-2800 \mathrm{kA} / \mathrm{m}$. This value of the critical external field is nearly independent of the damping constant. Then the magnetization reversal proceeds with different speeds. Once a reversed domain is formed it expands rapidly for high damping. Precessional motion slows down domain expansion for low damping.

Fig. 20 illustrates the magnetization reversal process of the perpendicular storage element. Reversed domains are formed at two opposite corners. The 


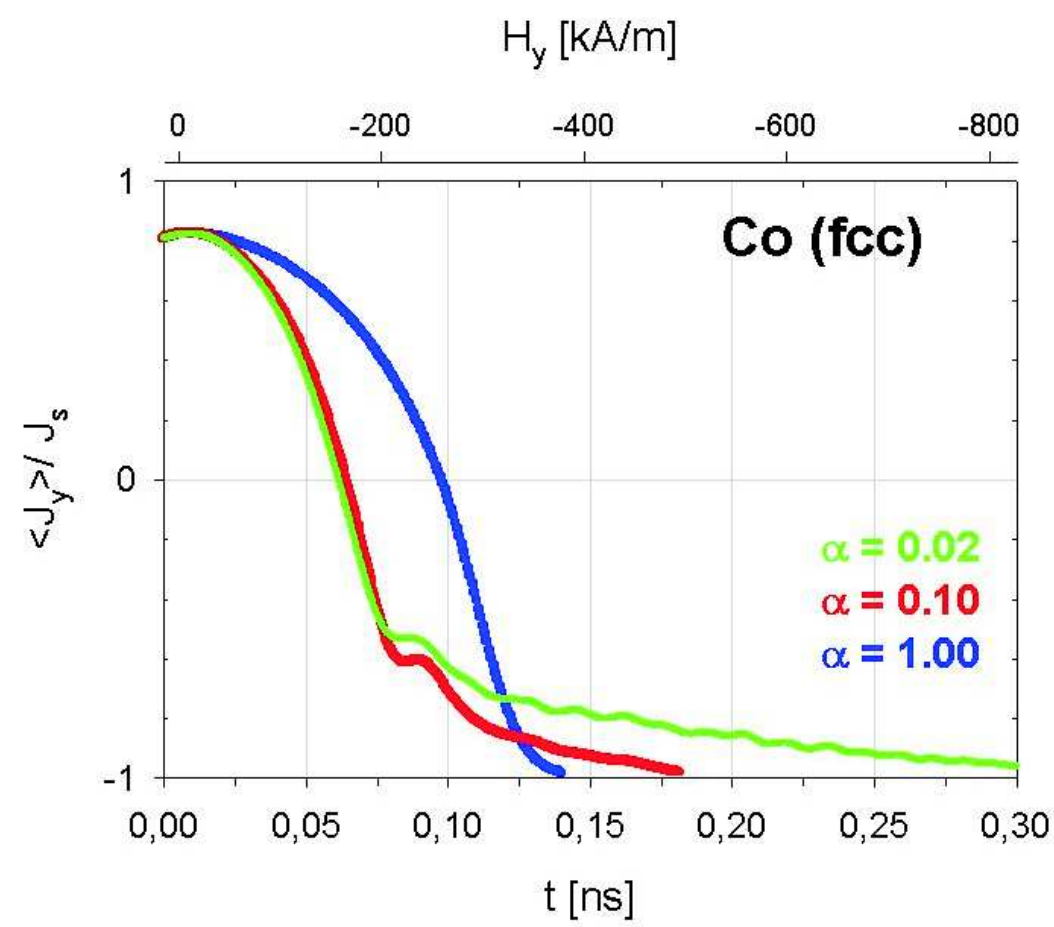

Fig. 13. Fast switching of polycrystalline fcc-Co. Time evolution of the magnetization component parallel to the direction of the applied field for three different values of the damping constant $\alpha$. The sweep rate of the applied field is $-2800 \mathrm{kA} /(\mathrm{m} \cdot \mathrm{ns})$.

reversed domains expand, merge, and form a channel of reversed magnetization. Finally, a large reversed domain is formed. The nucleation field that is required to form a reversed domain depends on the local demagnetizing field and the misalignment of the grains. The higher the sweep rate of the external field, the more reversed domains nucleate initially.

\section{Effects of non-zero temperature}

The deterministic LLG equation (10) is believed to describe the physical path of the magnetization towards equilibrium, taking into account gyromagnetic precession and damping. In real systems, thermal fluctuations change the deterministic motion of the magnetization into a random walk. A theoretical description must treat magnetization reversal as a stochastic process. The magnetic properties like the coercive field and the switching time follow from averages over many numerical realizations of the reversal process.

A stochastic equation which describes thermally activated magnetization reversal is the Langevin equation [32]. It follows from the LLG equation by adding a random thermal fluctation field to the effective magnetic field. 

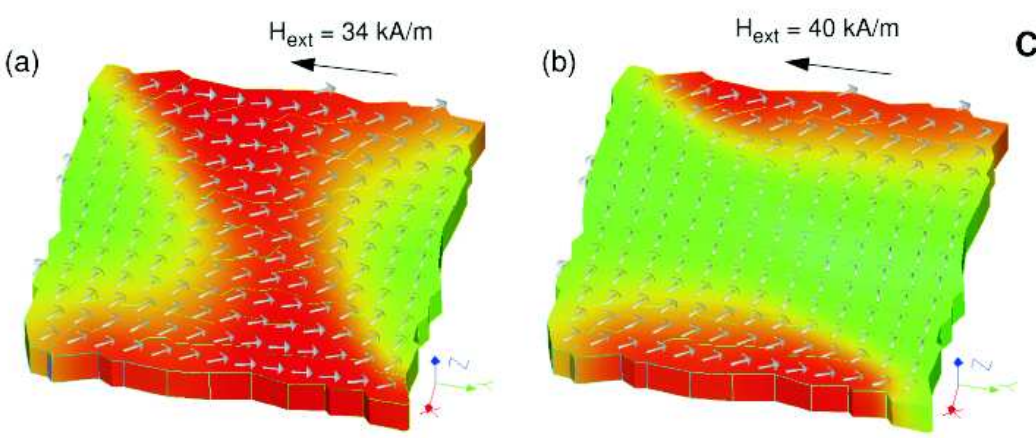

Co (fcc)
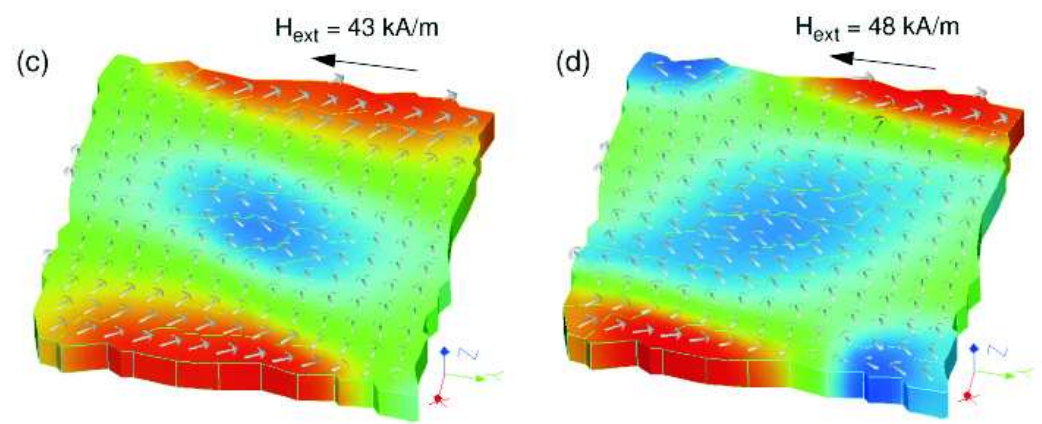

Fig. 14. Magnetization distribution of polycrystalline fcc-Co during the application of a unidirectional field with a sweep rate of $-280 \mathrm{kA} /(\mathrm{m} \cdot \mathrm{ns})$ and a Gilbert damping constant $\alpha=0.1$. The grey scale maps the magnetization component parallel to the field direction. (a) $\left\langle J_{y}\right\rangle / J_{\mathrm{s}}=0.6$, (b) $\left\langle J_{y}\right\rangle / J_{\mathrm{s}}=0.3$, (c) $\left\langle J_{y}\right\rangle / J_{\mathrm{s}}=0$, and (d) $\left\langle J_{y}\right\rangle / J_{\mathrm{s}}=-0.3$.

Garcia-Palacíos and Lázaro [33] numerically solved the Langevin equation for a single magnetic moment. They reported important phenomena like crossing-back or multiple crossing of the energy barrier which are attributed to the gyromagnetic nature of the system. As in the deterministic case the critical value of the damping constant that minimizes the switching time is $\alpha=1$ for uniform rotation [34]. Zhang and Fredkin [35] used the finite element method to study thermally activated reversal in ellipsoidal particles large enough to show an inhomogeneous reversal process.

\subsection{Langevin micromagnetics}

In order to treat thermally activated processes a stochastic thermal field, $\boldsymbol{H}_{\mathrm{th}}$, is added to the effective field, $\boldsymbol{H}_{\text {eff }}$. The thermal field is assumed to be a Gaussian random process with the following statistical properties:

$$
\begin{aligned}
\left\langle H_{\mathrm{th}, i}(\mathbf{r}, t)\right\rangle & =0 \\
\left\langle H_{\mathrm{th}, i}(\boldsymbol{r}, t) H_{\mathrm{th}, j}\left(\boldsymbol{r}^{\prime}, t^{\prime}\right)\right\rangle & =2 D \delta_{i j} \delta\left(\boldsymbol{r}-\boldsymbol{r}^{\prime}\right) \delta\left(t-t^{\prime}\right) .
\end{aligned}
$$




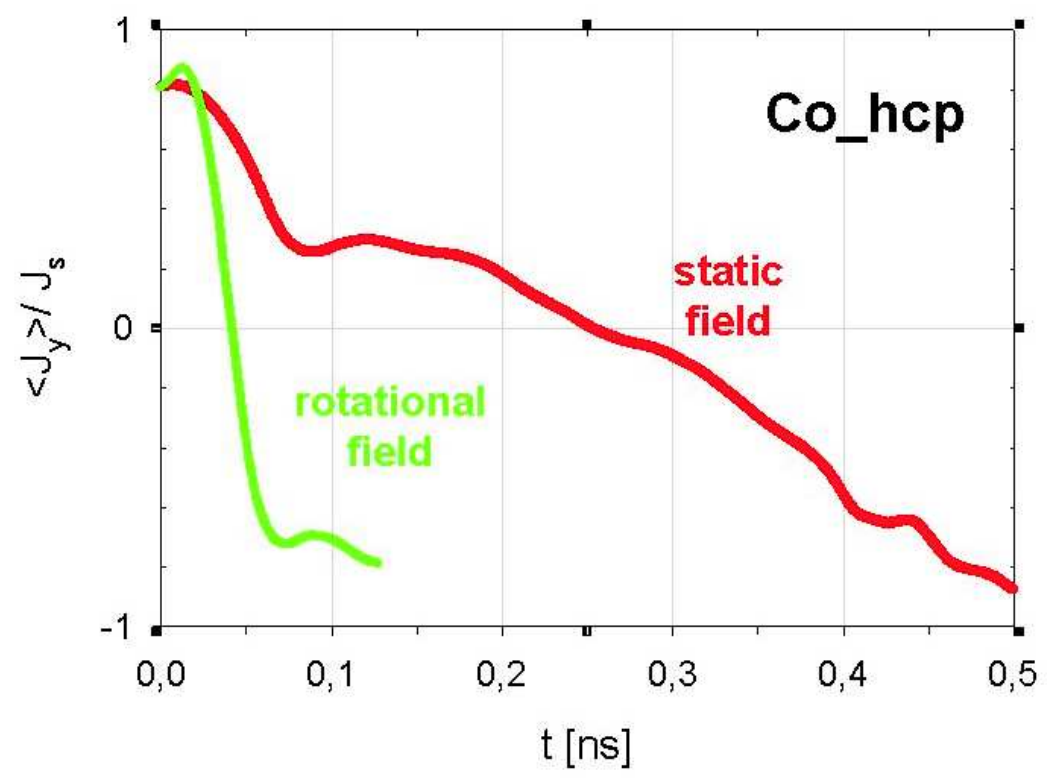

Fig. 15. Switching of an hcp-Co film in a constant unidirectional field and a rotational field. Time evolution of the magnetization for a unidirectional field of $H_{\text {ext }}=-140 \mathrm{kA} / \mathrm{m}$ and a rotational field of $\left|H_{\text {ext }}\right|=140 \mathrm{kA} / \mathrm{m}$ with a frequency of $10 \mathrm{GHz}$. The damping constant is $\alpha=0.1$.

(a)

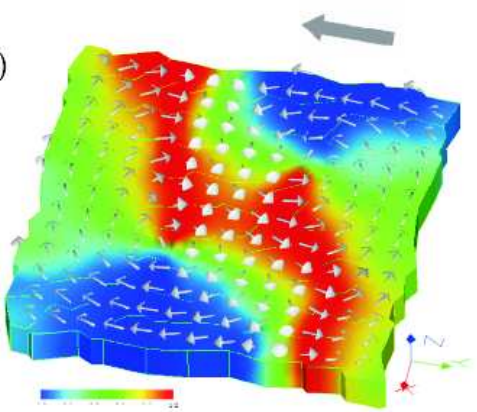

(b)

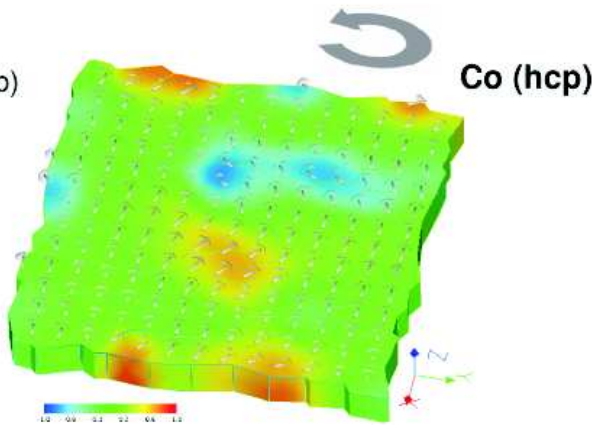

Fig. 16. Magnetization distribution of hcp-Co during the reversal under the influence of a unidirectional field and a rotational field. The Gilbert damping constant is $\alpha=0.1$. (a) Constant unidirectional field, $H_{\text {ext }}=-140 \mathrm{kA} / \mathrm{m}$ at $\left\langle J_{y}\right\rangle / J_{\mathrm{s}}=0$; (b) rotational field with a frequency of $10 \mathrm{GHz},\left|H_{\text {ext }}\right|=140 \mathrm{kA} / \mathrm{m}$ at $\left\langle J_{y}\right\rangle / J_{\mathrm{s}}=0$. 


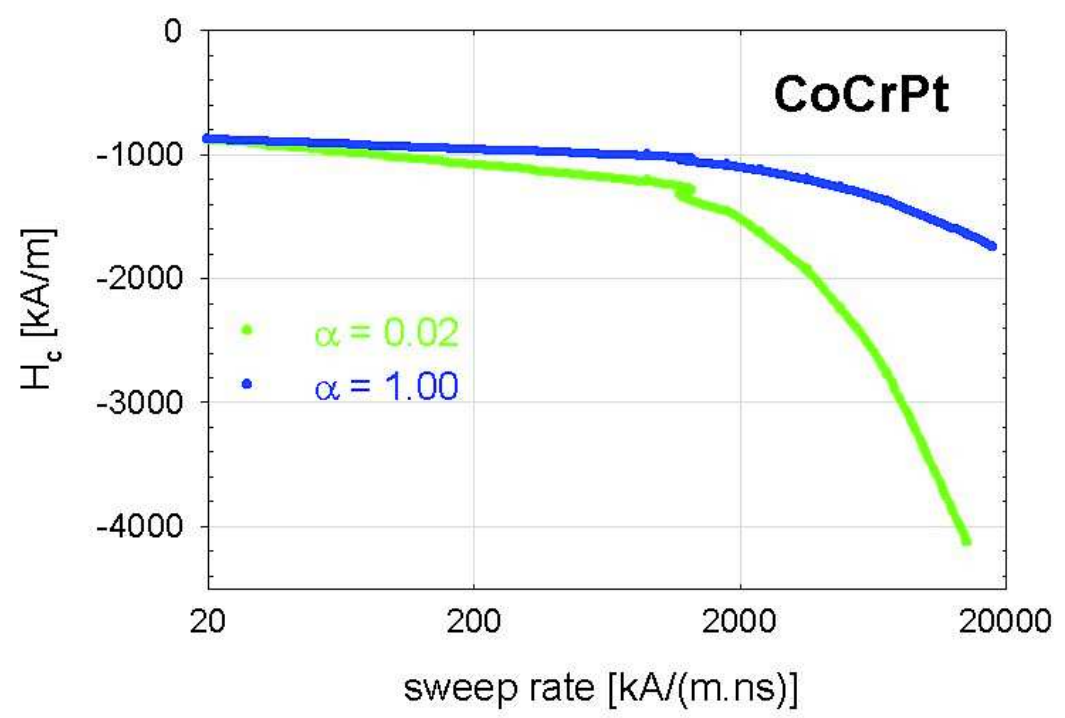

Fig. 17. Perpendicular storage element (CoCrPt). Dynamic coercivity as a function of the field sweep rate for two different values of the damping constant $\alpha$.

The average of the thermal field, taken over different realizations, vanishes in each direction $i$ in space. The thermal field is uncorrelated in time and space. The strength of the thermal fluctuations follow from the fluctuationdissipation theorem [36]:

$$
D=\frac{\alpha k_{\mathrm{B}} T}{\gamma \mu_{0}|\boldsymbol{m}|} .
$$

\subsection{Switching dynamics at non-zero temperature}

The fast switching experiments for small magnetic particles presented in section 3 were repeated at room temperature. The results show that thermal effects do not change the dependence of the switching time on the strength of the external field. The solid line in Fig. 3 shows the switching time at $T=300 \mathrm{~K}$ as a function of the field strength. The results at non-zero temperature have been averaged over 100 realizations of the stochastic switching process. Thermal fluctuations reduce the switching time. In the investigated temperature range $(0 \mathrm{~K}-400 \mathrm{~K})$ the switching time decreases almost linearly as a function of the temperature.

The effect of thermal fluctuations on the reversal process can be understood comparing the trajectories of the magnetic moment for $T=0$ and non-zero temperature. Fig. 21 shows the motion of the magnetic moment in the $x y$-plane at $T=0 \mathrm{~K}$ and $T=50 \mathrm{~K}$. Only the path during the first three precessional circles after the application of the external field is shown. 


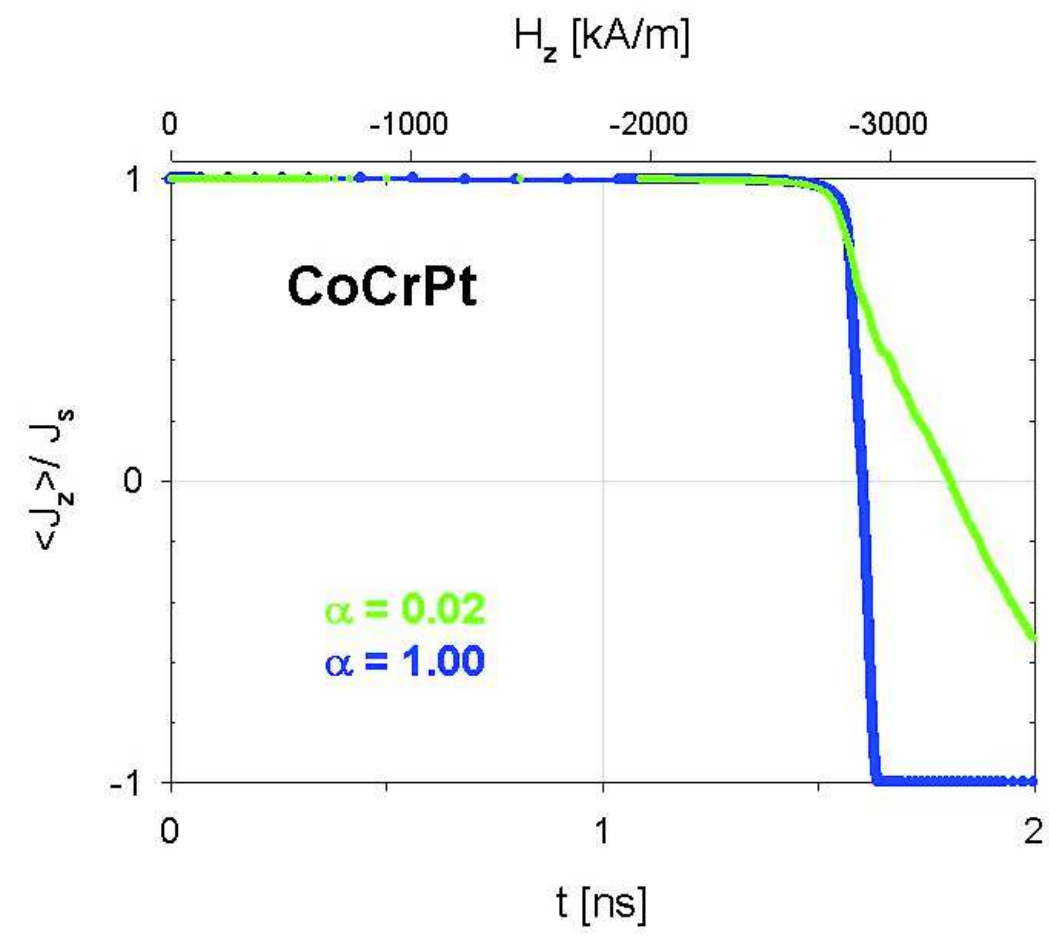

Fig. 18. Slow switching of a perpendicular storage element (CoCrPt). Time evolution of the magnetization component parallel to the direction of the applied field for two different values of the damping constant $\alpha$. The sweep rate of the applied field is $-1800 \mathrm{kA} /(\mathrm{m} \cdot \mathrm{ns})$.

The Gilbert damping constant for these simulations was $\alpha=0.1$. The radius of the precessional circles increases more rapidly at non-zero temperature. Therefore, at $T=50 \mathrm{~K}$ the number of precessional circles until $J_{\mathrm{z}}$ reaches zero is smaller than at $T=0$. This indicates that the system at $T=50 \mathrm{~K}$ is effectively stronger damped.

In order to explain this effect, let us assume that the system is in the state $\mathbf{S}$ as indicated by the black dot in Fig. 21. The thermal field causes fluctuations of the orientation of the magnetic moment. If the magnetic moment is perturbed into a state within the region $T_{1}$ this state is closer to the final state. Since the area $T_{1}$ is larger than the area $T_{2}$ thermal fluctuations favor the relaxation of the system.

This effective increase of the damping is most pronounced when the effective field points almost antiparallel to the magnetic moment. In this case thermal fluctuations always increase the angle between the magnetic moment and the effective field which causes a higher torque. With increasing angle between the magnetic moment and the effective field the effect vanishes. If 


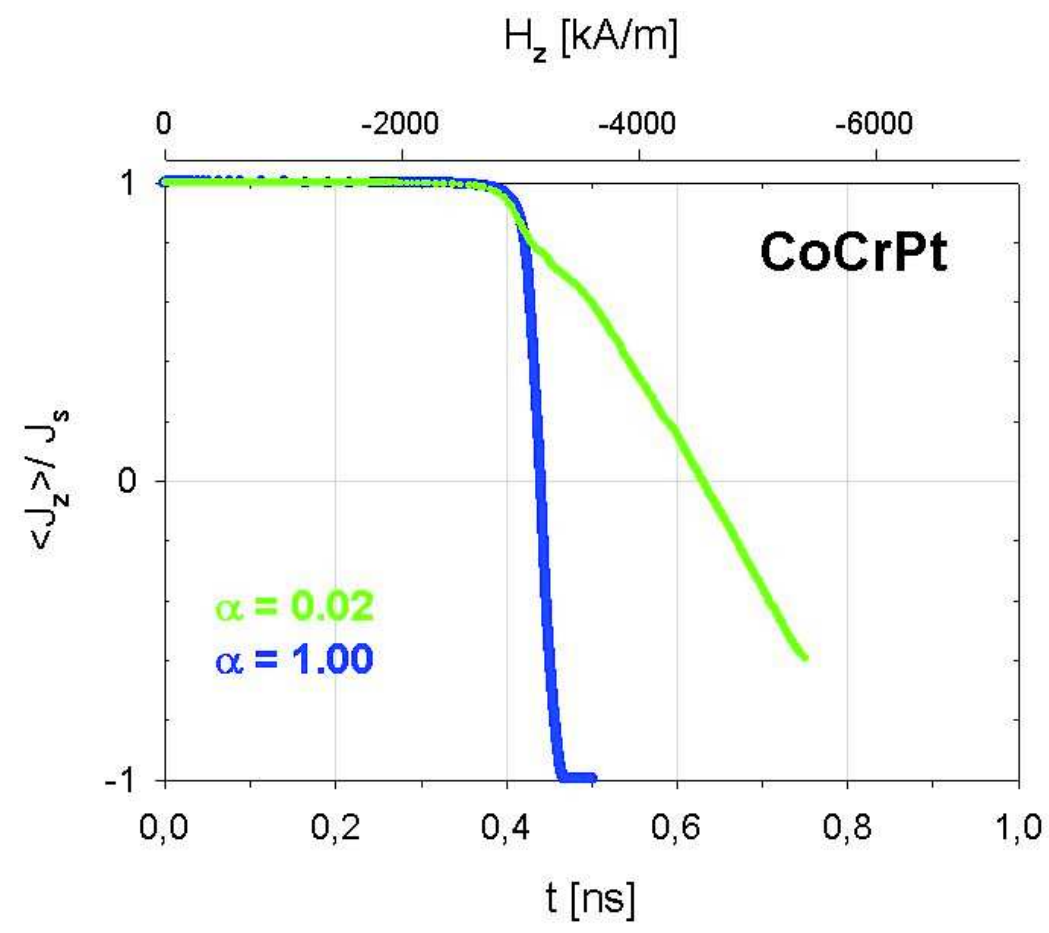

Fig. 19. Fast switching of a perpendicular storage element (CoCrPt). Time evolution of the magnetization component parallel to the direction of the applied field for two different values of the damping constant $\alpha$. The sweep rate of the applied field is $-7200 \mathrm{kA} /(\mathrm{m} \cdot \mathrm{ns})$.

the external field is for example applied $45^{\circ}$ off the easy axis the switching time does not significantly change with temperature.

In granular hcp-Co film thermally activated processes were found to reduce the switching time by less than $10 \%$ if the external field is in the order of the static coercivity. For large external field or high sweep rates dynamic processes override the thermal effects.

\section{Summary}

The anisotropy of the grains, and the orientation and strength of the applied field determine the switching speed of granular, mesoscopic magnets. Understanding and controlling the magnetic switching dynamics of magnetic particles is the major challenge for technological applications. Finite element micromagnetic simulations provide the details of the reversal process in uniaxial Co thin film elements, in cubic Co thin film elements, and in perpendicular $\mathrm{CoCrPt}$ storage elements. Magnetization reversal by nonuniform rotation is considerably faster than switching by the nucleation and expansion 
(a)

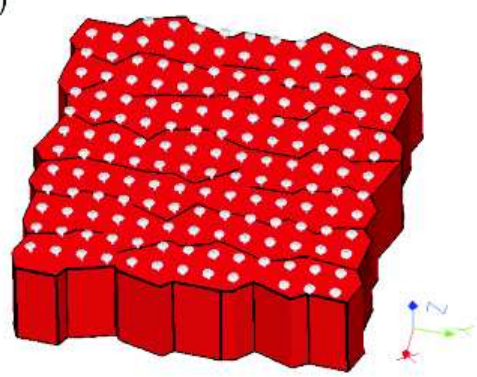

(c)

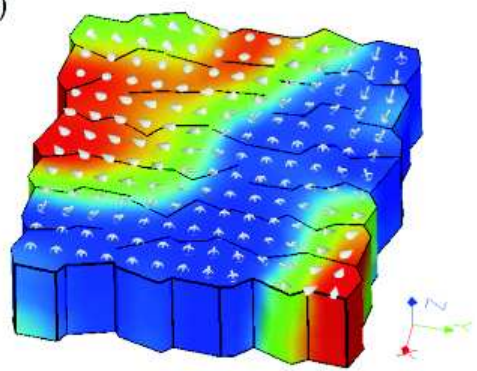

(b)

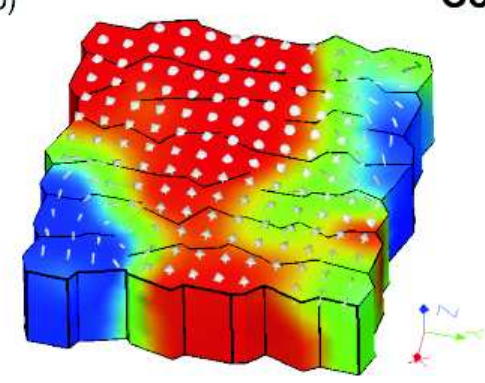

(d)

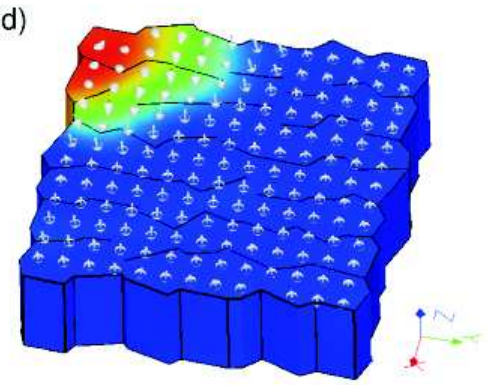

Fig. 20. Magnetization distribution of a perpendicular storage element ( $\mathrm{CoCrPt})$ during the application of a unidirectional field with a sweep rate of $-3600 \mathrm{kA} /(\mathrm{m} \cdot \mathrm{ns})$. The Gilbert damping constant $\alpha=0.02$. The grey scale maps the magnetization component parallel to the film normal. From (a) to (d) the perpendicular field decreases.

of reversed domains. Rotational switching is found in granular films with cubic magnetocrystalline anisotropy and can be achieved by apply a rotational external field in uniaxial materials. The dynamic coercivity increases with increasing sweep rate of the external field. Small values of the damping constant lead to shorter switching times in thin film elements with in plane anisotropy, whereas low damping increases the switching time in thin film elements with perpendicular anisotropy.

\section{Acknowledgements}

This work was supported by the Austrian Science Fund (Y132-PHY, 13260 TEC).

\section{References}

1. E. W. Pugh: Ferrite core memories that shaped an industry, IEEE Trans. Magn. 20, 1499-1502 (1984) 


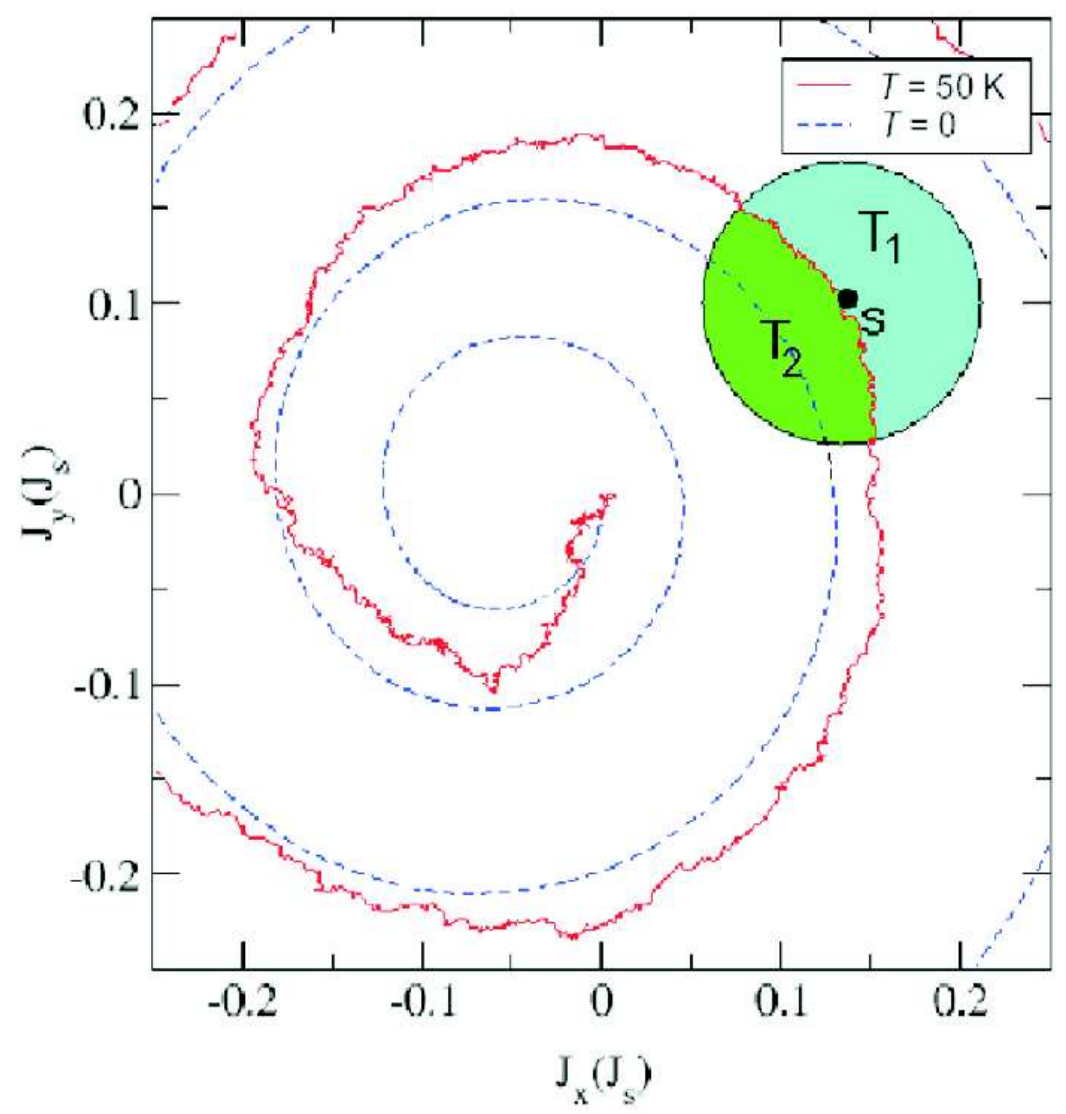

Fig. 21. Path of the magnetic moment of a small magnetic particle at $T=0$ and $T=50 \mathrm{~K}$. The trajectory is projected onto a plane perpendicular to the anisotropy axis. The external field of $H_{\text {ext }}=-1.5\left(2 \mathrm{~K} / J_{\mathrm{s}}\right)$ is applied $1^{\circ}$ off the anisotropy axis.

2. D. Weller, A. Moser: Thermal Effect Limits in Ultrahigh-Density Magnetic Recording, IEEE Trans. Magn. 35, 4423-4439 (1999)

3. R. H. Koch et al.: Magnetization reversal in micron-sized magnetic thin films, Phys. Rev. Lett. 81, 4512-4515 (1998)

4. D. Suess, T. Schrefl, J. Fidler: Reversal modes, thermal stability, and exchange length in perpendicular recording media, IEEE Trans Magn. 37, 1664-1666 (2001)

5. K. J. Kirk, J. N. Chapman, C. D. W. Wilkinson: Switching fields and magnetostatic interactions of thin film magnetic nanoelements, Appl. Phys. Lett. 71, 539-541 (1997)

6. C. H. Back, J. Heidmann, J. McCord: Time resolved Kerr microscopy: Magnetization dynamics in thin film write heads, IEEE Trans. Magn. 35, 637-642 (1999)

7. R. Kikuchi: On the minimum of magnetization reversal time, J. Appl. Phys. 27, 1352-1357 (1956) 
8. L. He, W. D. Doyle: A theoretical description of magnetic switching experiments in picosecond field pulses J. Appl. Phys. 79, 6489-6491 (1996)

9. M. Bauer, R. Lopusnik, J. Fassbinder, B. Hillebrands: Magnetization reversal in ultrashort magnetic field pulses, J. Magn. Magn. Mater. 218 165-176 (2000)

10. J. C. Mallinson: On damped gyromagnetic precession, IEEE Trans. Magn. 23, 2003-2004 (1987)

11. J. C. Mallinson: Damped gyromagnetic switching, IEEE Trans. Magn. 36, 1976-1981 (2000)

12. G. Albuquerque, J. Miltat, A. Thiaville: Coherent spin structures dynamics: numerics and application th high density magnetic random access memories (MRAMs), in , 16th IMACS World Congress On Scientific Computation, Applied Mathematics and Simulation, Lausanne, Switzerland, 2000, M. Deville, R. Owens (Eds.)

13. L. He, W. D. Doyle, L. Varga, H. Fujiwara, P. J. Flanders: High-Speed Switching in Magnetic Recording Media, J. Magn. Mater. 155, 6-12 (1996)

14. F. Akagi, A. Nakamura et al.: Computer Simulation of Magnetization Switching Behavior in High-Data-Rate Hard-Disk Media, IEEE. Trans. Magn. 36, 154-158 (2000)

15. R. W. Harrell: Orientation dependence of the dynamic coercivity of StonerWohlfarth particles. IEEE Trans. Magn. 37, 533-537 (2001)

16. T. L. Gilbert: A Lagrangian formulation of gyromagnetic equation of the magnetization field, Phys. Rev. 100, 1243 (1955)

17. L. Landau, E. Lifshitz: On the theory of the dispersion of magnetic permeability in ferromagnetic bodies, Physikalische Zeitschrift der Sowjetunion 8, 154-169 (1935)

18. R. Street, D.C. Crew: Fluctuation aftereffects in magnetic materials, IEEE Trans. Magn. 35 4407-4413 (1999)

19. E. C. Stoner, E. P. Wohlfarth: A mechanism of magnetic hysteresis in heterogeneous alloys, Philosophical Transaction of the Royal Society of London A240, 599-642, (1948)

20. J. Fassbinder: Magnetization dynamics investigated by time-resolved Kerr effect magnetometry, this volume

21. W.F. Brown, Jr.: Micromagnetics, (Wiley, New York, 1963)

22. Schrefl T., Forster H. et al.: Micromagnetic Simulation of Switching Events, in: Advances in Solid State Physics 41 Kramer, B. (Ed.) (Springer, Berlin, Heidelberg 2001) pp. 623-635

23. S. D. Cohen, A. C. Hindmarsh: CVODE, A Stiff/Nonstiff ODE Solver in C, Computers in Physics 10 138-143 (1996)

24. D. R. Fredkin, T. R. Koehler: Hybrid method for computing demagnetizing fields, IEEE Trans. Magn. 26 415-417 (1990)

25. M. Johnson: Magnetoelectronic memories last and last ..., IEEE Spectrum 37, 33-40 (2000)

26. G. A. Prinz: Magnetoelectronic applications, J. Magn. Magn. Mater. 200, 57-68 (1999)

27. R. P. Cowburn, M. E. Welland: Room temperature magnetic quantum cellular automata, Science 287, 1466-1458 (2000)

28. S. E. Russek, R. D. McMichael, M. J. Donahue, S. Kaka: High-speed switching and rotational dynamics in small magnetic thin-film devices, this volume 
29. W. Yang, D. N. Lambeth, D. E. Laughlin: Dependence of Co anisotropy constants on temperature, processing, and underlayer, J. Appl. Phys. 87, 6884-6886 (2000)

30. L. Holloway, H. Laidler: Thermal activation effects in CoCrPtTa media due to stacking faults, IEEE Trans. Magn. 37 1459-1461 (2001)

31. C. T. Rettner, M. E. Best, B. D. Terris: Patterning of Granular Magnetic Media with a Focused Ion Beam to Produce Single-Domain Islands at $>140 \mathrm{Gbit} / \mathrm{in}^{2}$, IEEE Trans. Magn. 37 1649-1651 (2001)

32. W. F. Brown, Jr.: Thermal fluctuations of a single-domain particle, Phys. Rev. 130, 1677-1686 (1963)

33. J. L. García-Palacios, F. J. Lázaro: Langevin-dynamics study of the dynamical properties of small magnetic particles, Phys. Rev. B 58, 14937-14958 (1998)

34. D. Hinzke und U. Nowak: Magnetization Switching in Nanowires: Monte Carlo Study with Fast Fourier Transformation for Dipolar Fields, J. Magn. Magn. Mat. 221, 365-372 (2000)

35. K. Zhang, D. R. Fredkin: Stochastic dynamic micromagnetic study of fine particles, J. Appl. Phys. 85 5208-5210 (1999)

36. N. G. van Kampen: Stochastic processes in physics and chemistry, (NorthHolland, Amsterdam, 1992) 


\section{Index}

boundary element method, 11

Co-fcc, 13

Co-hcp, 12

CoCrPt, 17

discrete media, 17

dynamic coercivity, 12, 14, 18

finite element method, 10

Gilbert equation, 3

gyromagnetic precession, 6

high speed switching, 5

Landau-Lifshitz-Gilbert equation, 4 Langevin equation, 19

magnetic recording, 17 magnetic scalar potential, 11 magnetization reversal dynamics, 1 magnetization reversal mode, 16 magnetocrystalline anisotropy, 9 micromagnetics, 1, 9 microstructure, 10

MRAM, 9

nano-element, 11

nucleation, 15

perpendicular anisotropy, 17

submicron-sized magnets, 1

thermal fluctuation, 19

thin film element, 11

uniform rotation, 15 